Supporting Information

\title{
Biomimetic Synthesis of Moschamine-Related Indole Alkaloids via Iron-Catalyzed Selectively Oxidative Radical Coupling
}

Kangjiang Liang, ${ }^{\dagger, \S}$ Jing Yang, ${ }^{\dagger}$ Xiaogang Tong, ${ }^{\dagger}$ Wenbin Shang, ${ }^{\dagger, \S}$ Zhiqiang Pan, ${ }^{\dagger}$ and Chengfeng Xia*,†,

${ }^{\dagger}$ State Key Laboratory of Phytochemistry and Plant Resources in West China, Kunming Institute of Botany, Chinese Academy of Sciences, Kunming 650201, Yunnan, China

${ }^{\ddagger}$ State Key Laboratory of Bioorganic \& Natural Products Chemistry, Shanghai Institute of Organic Chemistry, Chinese Academy of Sciences, Shanghai 200032, China

${ }^{\S}$ University of Chinese Academy of Sciences, Beijing 100049, China

\section{List of Supporting Information}

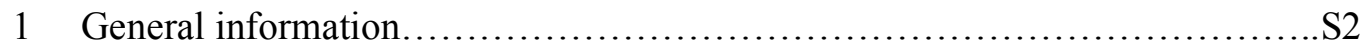

2 General procedures for iron-catalyzed oxidative radical coupling..............S2

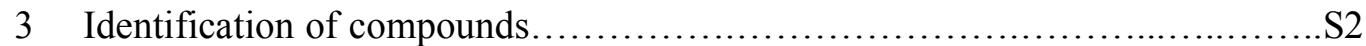

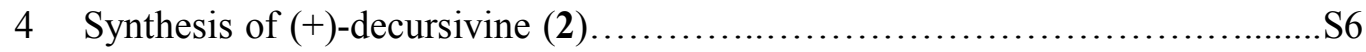

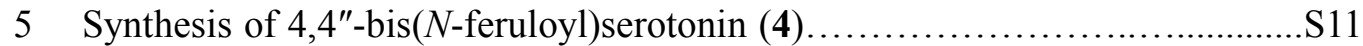

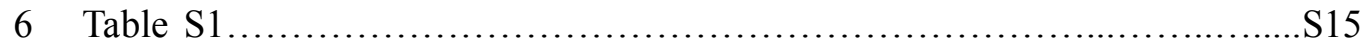

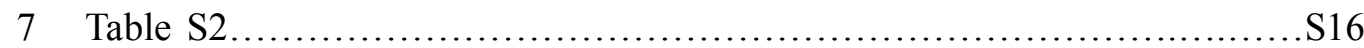

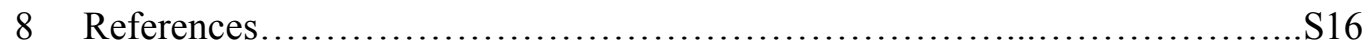

9 Copies of ${ }^{1} \mathrm{H}$ NMR and ${ }^{13} \mathrm{C}$ NMR spectra........................................... 17 


\section{General information}

${ }^{1} \mathrm{H}$ and ${ }^{13} \mathrm{C}$ NMR experiments were performed on a Bruker AM-400 and DRX-600 NMR spectrometer at ambient temperature. Chemical shifts (d) are expressed in p.p.m., and $J$-values are given in Hz. The residual solvent protons $\left({ }^{1} \mathrm{H}\right)$ or the solvent carbons $\left({ }^{13} \mathrm{C}\right)$ were used as internal standards. ESIMS and HRESIMS were taken on API STAR Pulsar. Reactions were performed under argon atmosphere using flame-dried glassware unless otherwise noted. All reagents were used as received without further purification unless indicated otherwise. Thin layer chromatographies were carried out on GF254 plates. Flash chromatography was performed with 300-400 mesh silica gels. Visualization of the developed chromatogram was performed by fluorescence quenching or by ceric ammonium molybdate, or $\mathrm{KMnO}_{4}$ stain. Yields reported were for isolated, spectroscopically pure compounds.

General procedures for iron-catalyzed oxidative coupling between 5-hydroxyindoles and styrenes

To an oven dried Schlenk tube equipped with a stir-bar, 5-hydroxyindoles $(0.10$ $\mathrm{mmol}), \mathrm{FeCl}_{3}(0.01 \mathrm{mmol})$ were added in the glovebox. Then the reaction tube was allowed to be vacuumed and purged with nitrogen for three times. DCM (3 mL) and styrenes $(1.00 \mathrm{mmol})$ were added simultaneously under nitrogen. DDQ $(0.12 \mathrm{mmol}$, $0.1 \mathrm{M}$ in $\mathrm{DCM} /$ toluene $=1: 1$ ) was added dropwise over $2 \mathrm{~h}$. The mixture was quenched with water and extracted with EtOAc $(15 \mathrm{~mL} \times 3)$. The combined organic layers were washed with brine, dried over anhydrous $\mathrm{Na}_{2} \mathrm{SO}_{4}$ and concentrated. The crude product was subjected to column chromatography on silica gel (petroleum ether/acetone $=5: 1$ ) to afford the product.

\section{Identification of compounds}

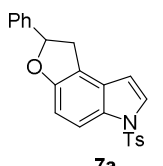

Compound 7a. White foam in $36 \mathrm{mg}, 92 \%$ yield. ${ }^{1} \mathrm{H}$ NMR (400 $\mathrm{MHz}, \mathrm{CDCl}_{3}$ ): $\delta$ $7.73(\mathrm{~d}, J=8.8,1 \mathrm{H}), 7.67(\mathrm{~d}, J=8.3,2 \mathrm{H}), 7.49(\mathrm{~d}, J=3.6,1 \mathrm{H}), 7.35-7.19(\mathrm{~m}, 5 \mathrm{H})$, $7.13(\mathrm{~d}, J=8.3,2 \mathrm{H}), 6.81(\mathrm{~d}, J=8.8,1 \mathrm{H}), 6.38(\mathrm{~d}, J=3.6,1 \mathrm{H}), 5.74(\mathrm{t}, J=8.8,1 \mathrm{H})$, $3.64(\mathrm{dd}, J=15.7,9.6,1 \mathrm{H}), 3.21(\mathrm{dd}, J=15.7,8.1,1 \mathrm{H}), 2.27(\mathrm{~s}, 3 \mathrm{H}),{ }^{13} \mathrm{C}$ NMR $(100$ $\left.\mathrm{MHz}, \mathrm{CDCl}_{3}\right): \delta 156.3,144.9,142.0,135.2,130.3,129.9,128.6,128.1,128.0,127,9$, 126.9, 125.7, 116.7, 113.0, 107.0, 106.8, 84.4, 37.5, 21.6; HR-ESI-MS (m/z): calcd. for $\mathrm{C}_{23} \mathrm{H}_{20} \mathrm{NO}_{3} \mathrm{~S}[\mathrm{M}+\mathrm{H}]^{+}, 390.1158$, found 390.1155 . 


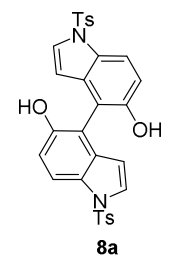

Compound 8a. White foam in $4 \mathrm{mg}, 15 \%$ yield. ${ }^{1} \mathrm{H}$ NMR (400 $\left.\mathrm{MHz}, \mathrm{CDCl}_{3}\right): \delta 7.89$ $(\mathrm{d}, J=8.9,2 \mathrm{H}), 7.71(\mathrm{~d}, J=8.3,4 \mathrm{H}), 7.42(\mathrm{~d}, J=3.6,2 \mathrm{H}), 7.19(\mathrm{~d}, J=8.3,4 \mathrm{H}), 6.98$ $(\mathrm{d}, J=8.9,2 \mathrm{H}), 6.01(\mathrm{~d}, J=3.6,2 \mathrm{H}), 4.95(\mathrm{~s}, 2 \mathrm{H}), 2.30(\mathrm{~s}, 6 \mathrm{H}) ;{ }^{13} \mathrm{C} \mathrm{NMR}(100 \mathrm{MHz}$, $\left.\mathrm{CDCl}_{3}\right) \delta 149.8,145.2,135.2,131.1,130.0,129.6,127.5,126.9,115.2,114.5,110.8$, 108.2, 21.6; HR-ESI-MS (m/z): calcd. for $\mathrm{C}_{30} \mathrm{H}_{25} \mathrm{~N}_{2} \mathrm{O}_{6} \mathrm{~S}_{2}[\mathrm{M}+\mathrm{H}]^{+}, 573.1149$, found 573.1140 .

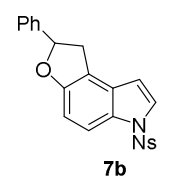

Compound 7b. Yellow foam in $5 \mathrm{mg}, 12 \%$ yield. ${ }^{1} \mathrm{H}$ NMR (400 $\mathrm{MHz}, \mathrm{CDCl}_{3}$ ): $\delta$ $7.82(\mathrm{~d}, J=8.0,1 \mathrm{H}), 7.74-7.61(\mathrm{~m}, 5 \mathrm{H}), 7.44-7.28(\mathrm{~m}, 5 \mathrm{H}), 6.90(\mathrm{~d}, J=8.8,1 \mathrm{H})$, $6.56(\mathrm{~d}, J=3.7,1 \mathrm{H}), 5.98-5.73(\mathrm{~m}, 1 \mathrm{H}), 3.78(\mathrm{dd}, J=15.8,9.7,1 \mathrm{H}), 3.34(\mathrm{dd}, J=15.8$, 8.0, $1 \mathrm{H}) ;{ }^{13} \mathrm{C} \mathrm{NMR}\left(100 \mathrm{MHz}, \mathrm{CDCl}_{3}\right): \delta 156.7,141.9,134.8,132.3,131.8,129.9$, $128.9,128.7,128.1,128.0,125.7,124.9,117.3,112.8,107.2,106.6,84.5,37.5$; HR-ESI-MS $(m / z)$ : calcd. for $\mathrm{C}_{22} \mathrm{H}_{17} \mathrm{~N}_{2} \mathrm{O}_{5} \mathrm{~S}[\mathrm{M}+\mathrm{H}]^{+}$, 421.0853, found 421.0849.

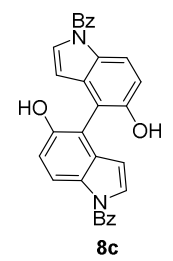

Compound 8c. White foam in $6 \mathrm{mg}, 26 \%$ yield. ${ }^{1} \mathrm{H}$ NMR (400 MHz, acetone- $\left.d_{6}\right): \delta$ $8.31(\mathrm{~d}, J=8.8,2 \mathrm{H}), 7.96(\mathrm{~s}, 2 \mathrm{H}), 7.79(\mathrm{~d}, J=7.1,4 \mathrm{H}), 7.67-7.58(\mathrm{~m}, 6 \mathrm{H}), 7.28(\mathrm{~d}, J$ $=3.7,2 \mathrm{H}), 7.13(\mathrm{~d}, J=8.8,2 \mathrm{H}), 6.18(\mathrm{~d}, J=3.7,2 \mathrm{H}) ;{ }^{13} \mathrm{C}$ NMR $(150 \mathrm{MHz}$, acetone- $\left.d_{6}\right): \delta 168.9,152.4,135.8,133.0,132.5,131.1,129.7,129.4,128.9,117.4$, 115.1, 114.6, 109.3; HR-ESI-MS (m/z): calcd. for $\mathrm{C}_{30} \mathrm{H}_{21} \mathrm{~N}_{2} \mathrm{O}_{4}[\mathrm{M}+\mathrm{H}]^{+}, 473.1496$, found 473.1498 .

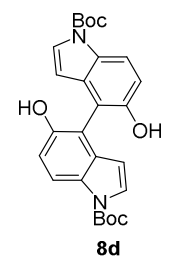

Compound 8d. Colorless oil in $11 \mathrm{mg}, 45 \%$ yield. ${ }^{1} \mathrm{H}$ NMR (400 $\left.\mathrm{MHz}, \mathrm{CDCl}_{3}\right): \delta$ 
$8.16(\mathrm{~d}, J=8.4,2 \mathrm{H}), 7.54(\mathrm{~d}, J=3.6,2 \mathrm{H}), 7.10(\mathrm{~d}, J=8.9,2 \mathrm{H}), 6.11(\mathrm{~d}, J=3.6,2 \mathrm{H})$, $5.02(\mathrm{~s}, 2 \mathrm{H}), 1.68(\mathrm{~s}, 18 \mathrm{H}) ;{ }^{13} \mathrm{C} \mathrm{NMR}\left(100 \mathrm{MHz}, \mathrm{CDCl}_{3}\right): \delta 165.1,149.6,149.5$, $130.8,127.1,116.9,113.8,110.4,106.4,83.8,28.2$; HR-ESI-MS $(\mathrm{m} / \mathrm{z})$ : calcd. for $\mathrm{C}_{26} \mathrm{H}_{29} \mathrm{~N}_{2} \mathrm{O}_{6}[\mathrm{M}+\mathrm{H}]^{+}, 465.2020$, found 465.2023.

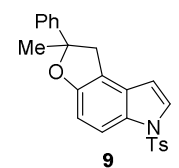

Compound 9. White foam in $33 \mathrm{mg}, 82 \%$ yield. ${ }^{1} \mathrm{H}$ NMR (400 MHz, $\mathrm{CDCl}_{3}$ ): $\delta 7.80$ $(\mathrm{d}, J=8.8,1 \mathrm{H}), 7.74(\mathrm{~d}, J=8.3,2 \mathrm{H}), 7.54(\mathrm{~d}, J=3.7,1 \mathrm{H}), 7.48(\mathrm{~d}, J=7.5,2 \mathrm{H}), 7.33$ (t, $J=7.6,2 \mathrm{H}), 7.28-7.18(\mathrm{~m}, 3 \mathrm{H}), 6.92(\mathrm{~d}, J=8.8,1 \mathrm{H}), 6.43(\mathrm{~d}, J=3.6,1 \mathrm{H})$, 3.57-3.41 (m, 2H), $2.33(\mathrm{~s}, 3 \mathrm{H}), 1.79(\mathrm{~s}, 3 \mathrm{H}) ;{ }^{13} \mathrm{C} \mathrm{NMR}\left(100 \mathrm{MHz}, \mathrm{CDCl}_{3}\right): \delta 155.5$, $146.9,144.8,135.2,130.2,129.8,128.4,128.1,127.7,127.1,126.8,124.5,116.6$, 113.0, 107.2, 106.8, 89.6, 43.9, 29.5, 21.6; HR-ESI-MS $(\mathrm{m} / \mathrm{z})$ : calcd. for $\mathrm{C}_{24} \mathrm{H}_{22} \mathrm{NO}_{3} \mathrm{~S}$ $[\mathrm{M}+\mathrm{H}]^{+}, 404.1315$, found 404.1308 .

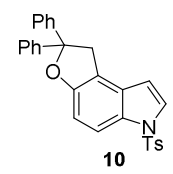

Compound 10. White foam in $45 \mathrm{mg}, 96 \%$ yield. ${ }^{1} \mathrm{H}$ NMR (400 $\mathrm{MHz}, \mathrm{CDCl}_{3}$ ): $\delta$ $7.72(\mathrm{~d}, J=8.8,1 \mathrm{H}), 7.64(\mathrm{~d}, J=8.3,2 \mathrm{H}), 7.46(\mathrm{~d}, J=3.6,1 \mathrm{H}), 7.40(\mathrm{~d}, J=7.5,4 \mathrm{H})$, $7.21(\mathrm{t}, J=7.5,4 \mathrm{H}), 7.18-7.08(\mathrm{~m}, 4 \mathrm{H}), 6.89(\mathrm{~d}, J=8.8,1 \mathrm{H}), 6.37(\mathrm{~d}, J=3.6,1 \mathrm{H})$, $3.91(\mathrm{~s}, 2 \mathrm{H}), 2.23(\mathrm{~s}, 3 \mathrm{H}) ;{ }^{13} \mathrm{C} \mathrm{NMR}\left(100 \mathrm{MHz}, \mathrm{CDCl}_{3}\right): \delta 156.3,145.5,144.8,135.2$, $130.4,129.9,128.3,127.9,127.8,127.4,126.8,126.0,116.5,113.1,107.2,106.8$, 92.3, 43.6, 21.6; HR-ESI-MS $(\mathrm{m} / \mathrm{z})$ : calcd. for $\mathrm{C}_{29} \mathrm{H}_{24} \mathrm{NO}_{3} \mathrm{~S}[\mathrm{M}+\mathrm{H}]^{+}, 466.1471$, found 466.1460 .

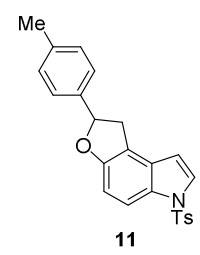

Compound 11. White foam in $31 \mathrm{mg}, 77 \%$ yield. ${ }^{1} \mathrm{H}$ NMR (400 $\left.\mathrm{MHz}, \mathrm{CDCl}_{3}\right): \delta$ $7.79(\mathrm{~d}, J=8.8,1 \mathrm{H}), 7.74(\mathrm{~d}, J=8.3,2 \mathrm{H}), 7.55(\mathrm{~d}, J=3.6,1 \mathrm{H}), 7.28(\mathrm{~d}, J=8.0,2 \mathrm{H})$, $7.21(\mathrm{~d}, J=8.1,2 \mathrm{H}), 7.15(\mathrm{~d}, J=7.9,2 \mathrm{H}), 6.87(\mathrm{~d}, J=8.8,1 \mathrm{H}), 6.45(\mathrm{~d}, J=3.6,1 \mathrm{H})$, $5.77(\mathrm{t}, J=8.8,1 \mathrm{H}), 3.67(\mathrm{dd}, J=15.7,9.6,1 \mathrm{H}), 3.26(\mathrm{dd}, J=15.7,8.2,1 \mathrm{H}), 2.34(\mathrm{~s}$, $3 \mathrm{H}), 2.33(\mathrm{~s}, 3 \mathrm{H}) ;{ }^{13} \mathrm{C} \mathrm{NMR}\left(100 \mathrm{MHz}, \mathrm{CDCl}_{3}\right): \delta 156.3,144.8,138.9,137.8,135.2$, 130.2, 129.9, 129.3, 128.0, 127.9, 126.8, 125.8, 116.9, 113.0, 107.0, 106.8, 84.4, 37.5, 21.6, 21.2; HR-ESI-MS $(\mathrm{m} / \mathrm{z})$ : calcd. for $\mathrm{C}_{24} \mathrm{H}_{22} \mathrm{NO}_{3} \mathrm{~S}[\mathrm{M}+\mathrm{H}]^{+}$, 404.1315, found 


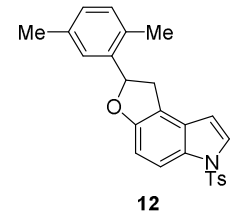

Compound 12. White foam in $36 \mathrm{mg}, 86 \%$ yield. ${ }^{1} \mathrm{H}$ NMR (400 $\left.\mathrm{MHz}, \mathrm{CDCl}_{3}\right)$ : $\delta$ $7.81(\mathrm{~d}, J=8.8,1 \mathrm{H}), 7.75(\mathrm{~d}, J=8.2,2 \mathrm{H}), 7.55(\mathrm{~d}, J=3.6,1 \mathrm{H}), 7.27-7.19(\mathrm{~m}, 3 \mathrm{H})$, $7.06(\mathrm{~d}, J=7.7,1 \mathrm{H}), 6.99(\mathrm{~d}, J=7.6,1 \mathrm{H}), 6.92(\mathrm{~d}, J=8.8,1 \mathrm{H}), 6.44(\mathrm{~d}, J=3.6,1 \mathrm{H})$, $5.96(\mathrm{t}, J=9.0,1 \mathrm{H}), 3.72(\mathrm{dd}, J=15.6,9.7,1 \mathrm{H}), 3.13(\mathrm{dd}, J=15.6,8.4,1 \mathrm{H}), 2.34(\mathrm{~s}$, $3 \mathrm{H}), 2.31(\mathrm{~s}, 3 \mathrm{H}), 2.26(\mathrm{~s}, 3 \mathrm{H}) ;{ }^{13} \mathrm{C} \mathrm{NMR}\left(100 \mathrm{MHz}, \mathrm{CDCl}_{3}\right): \delta 156.4,144.8,139.9$, $135.8,135.2,130.9,130.5,130.6,129.9,128.3,128.1,127.8,126.8,125.5,116.7$, 113.0, 107.0, 106.8, 82.0, 36.7, 21.6, 21.1, 18.8; HR-ESI-MS $(\mathrm{m} / \mathrm{z})$ : calcd. for $\mathrm{C}_{25} \mathrm{H}_{24} \mathrm{NO}_{3} \mathrm{~S}[\mathrm{M}+\mathrm{H}]^{+}, 418.1471$, found 418.1467 .

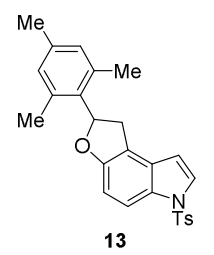

Compound 13. White foam in $24 \mathrm{mg}, 55 \%$ yield. ${ }^{1} \mathrm{H}$ NMR (400 $\mathrm{MHz}, \mathrm{CDCl}_{3}$ ): 7.80 $(\mathrm{d}, J=8.8,1 \mathrm{H}), 7.76(\mathrm{~d}, J=8.3,2 \mathrm{H}), 7.57(\mathrm{~d}, J=3.6,1 \mathrm{H}), 7.23(\mathrm{~d}, J=8.2,2 \mathrm{H}), 6.83$ (m, 3H), 6.47 (d, $J=3.6,1 \mathrm{H}), 6.16(\mathrm{t}, J=10.6,1 \mathrm{H}), 3.53(\mathrm{dd}, J=15.8,10.1,1 \mathrm{H})$, $3.31(\mathrm{dd}, J=15.8,11.1,1 \mathrm{H}), 2.35(\mathrm{~s}, 3 \mathrm{H}), 2.27(\mathrm{~s}, 6 \mathrm{H}), 2.26(\mathrm{~s}, 3 \mathrm{H}) ;{ }^{13} \mathrm{C}$ NMR $(100$ $\left.\mathrm{MHz}, \mathrm{CDCl}_{3}\right): \delta 156.2,144.8,137.5,136.7,135.3,133.2,130.1,130.0,129.9,127.8$, $127.7,126.8,117.4,113.2,107.3,106.8,82.0,35.1,21.6,20.8,20.7$; HR-ESI-MS $(\mathrm{m} / \mathrm{z})$ : calcd. for $\mathrm{C}_{26} \mathrm{H}_{26} \mathrm{NO}_{3} \mathrm{~S}[\mathrm{M}+\mathrm{H}]^{+}, 432.1628$, found 432.1624 .

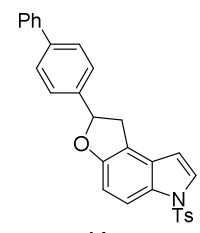

14

Compound 14. White foam in $19 \mathrm{mg}, 40 \%$ yield. ${ }^{1} \mathrm{H}$ NMR (400 $\mathrm{MHz}, \mathrm{CDCl}_{3}$ ): 7.81 $(\mathrm{d}, J=8.8,1 \mathrm{H}), 7.75(\mathrm{~d}, J=8.3,2 \mathrm{H}), 7.60-7.54(\mathrm{~m}, 5 \mathrm{H}), 7.48-7.39(\mathrm{~m}, 4 \mathrm{H}), 7.34(\mathrm{t}, J$ $=7.3,1 \mathrm{H}), 7.22(\mathrm{~d}, J=8.1,2 \mathrm{H}), 6.90(\mathrm{~d}, J=8.8,1 \mathrm{H}), 6.47(\mathrm{~d}, J=3.6,1 \mathrm{H}), 5.86(\mathrm{t}, J$ $=8.8,1 \mathrm{H}), 3.74(\mathrm{dd}, J=15.7,9.6,1 \mathrm{H}), 3.33(\mathrm{dd}, J=15.7,8.0,1 \mathrm{H}), 2.34(\mathrm{~s}, 3 \mathrm{H}) ;{ }^{13} \mathrm{C}$ NMR $\left(100 \mathrm{MHz}, \mathrm{CDCl}_{3}\right): \delta 156.3,144.9,141.1,141.0,140.7,135.2,130.3,129.9$, 128.8, 128.0, 127.9, 127.4, 127.1, 126.8, 126.2, 116.7, 113.1, 107.1, 106.8, 84.2, 37.5, 21.6; HR-ESI-MS $(m / z)$ : calcd. for $\mathrm{C}_{29} \mathrm{H}_{24} \mathrm{NO}_{3} \mathrm{~S}[\mathrm{M}+\mathrm{H}]^{+}, 466.1471$, found 466.1469 . 


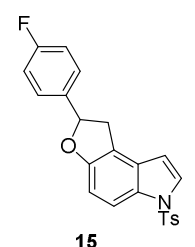

Compound 15. White foam in $29 \mathrm{mg}, 70 \%$ yield. ${ }^{1} \mathrm{H}$ NMR (400 $\mathrm{MHz}, \mathrm{CDCl}_{3}$ ): 7.80 $(\mathrm{d}, J=8.8,1 \mathrm{H}), 7.74(\mathrm{~d}, J=8.3,2 \mathrm{H}), 7.56(\mathrm{~d}, J=3.6,1 \mathrm{H}), 7.35(\mathrm{dd}, J=8.4,5.4,2 \mathrm{H})$, $7.21(\mathrm{~d}, J=8.1,2 \mathrm{H}), 7.02(\mathrm{t}, J=8.6,2 \mathrm{H}), 6.87(\mathrm{~d}, J=8.8,1 \mathrm{H}), 6.45(\mathrm{~d}, J=3.6,1 \mathrm{H})$, $5.78(\mathrm{t}, J=8.8,1 \mathrm{H}), 3.69(\mathrm{dd}, J=15.7,9.6,1 \mathrm{H}), 3.23(\mathrm{dd}, J=15.7,8.1,1 \mathrm{H}), 2.33$ (s, $3 \mathrm{H}) ;{ }^{13} \mathrm{C} \mathrm{NMR}\left(100 \mathrm{MHz}, \mathrm{CDCl}_{3}\right): \delta 163.7,161.3,156.1,144.9,137.8,137.7,135.2$, $130.3,129.9,128.0,127.9,127.6,127.5,126.8,116.5,115.6,115.4,113.1,107.0$, 106.7, 83.7, 37.6, 21.6; HR-ESI-MS $(\mathrm{m} / \mathrm{z})$ : calcd. for $\mathrm{C}_{23} \mathrm{H}_{19} \mathrm{FNO}_{3} \mathrm{~S}[\mathrm{M}+\mathrm{H}]^{+}$, 408.1064, found 408.1068.

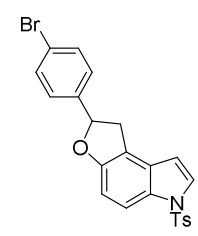

16

Compound 16. White foam in $30 \mathrm{mg}, 65 \%$ yield. ${ }^{1} \mathrm{H}$ NMR (400 $\mathrm{MHz}, \mathrm{CDCl}_{3}$ ): 7.80 $(\mathrm{d}, J=8.8,1 \mathrm{H}), 7.74(\mathrm{~d}, J=8.3,2 \mathrm{H}), 7.56(\mathrm{~d}, J=3.6,1 \mathrm{H}), 7.46(\mathrm{~d}, J=8.4,2 \mathrm{H}), 7.25$ $(\mathrm{d}, J=8.3,2 \mathrm{H}), 7.21(\mathrm{~d}, J=8.1,2 \mathrm{H}), 6.88(\mathrm{~d}, J=8.8,1 \mathrm{H}), 6.44(\mathrm{~d}, J=3.6,1 \mathrm{H}), 5.75$ (t, $J=8.8,1 \mathrm{H}), 3.70(\mathrm{dd}, J=15.7,9.7,1 \mathrm{H}), 3.20(\mathrm{dd}, J=15.7,7.9,1 \mathrm{H}), 2.33(\mathrm{~s}, 3 \mathrm{H})$; ${ }^{13} \mathrm{C}$ NMR $\left(100 \mathrm{MHz}, \mathrm{CDCl}_{3}\right): \delta 156.1,144.9,141.1,135.2,131.7,130.3,129.9$, 128.0, 127.4, 126.8, 121.8, 116.4, 113.1, 107.0, 106.7, 83.6, 37.5, 21.6; HR-ESI-MS $(\mathrm{m} / \mathrm{z})$ : calcd. for $\mathrm{C}_{23} \mathrm{H}_{19} \mathrm{BrNO}_{3} \mathrm{~S}[\mathrm{M}+\mathrm{H}]^{+}, 468.0264$, found 468.0272 .

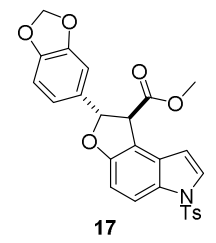

Compound 17. White foam in $33 \mathrm{mg}, 67 \%$ yield. ${ }^{1} \mathrm{H}$ NMR (400 $\mathrm{MHz}, \mathrm{CDCl}_{3}$ ): $\delta$ $7.88(\mathrm{~d}, J=8.9,1 \mathrm{H}), 7.76(\mathrm{~d}, J=8.3,2 \mathrm{H}), 7.58(\mathrm{~d}, J=3.6,1 \mathrm{H}), 7.25(\mathrm{~d}, J=8.3,2 \mathrm{H})$, $6.90(\mathrm{~d}, J=8.9,1 \mathrm{H}), 6.87-6.79(\mathrm{~m}, 2 \mathrm{H}), 6.76(\mathrm{~d}, J=7.9,1 \mathrm{H}), 6.62(\mathrm{~d}, J=3.6,1 \mathrm{H})$, $6.07(\mathrm{~d}, J=6.7,1 \mathrm{H}), 5.94(\mathrm{~s}, 2 \mathrm{H}), 4.37(\mathrm{~d}, J=6.7,1 \mathrm{H}), 3.79(\mathrm{~s}, 3 \mathrm{H}), 2.36(\mathrm{~s}, 3 \mathrm{H})$; ${ }^{13} \mathrm{C}$ NMR $\left(100 \mathrm{MHz}, \mathrm{CDCl}_{3}\right): \delta 171.4,156.1,148.1,147.7,145.0,135.2,134.6$, $130.5,129.9,128.0,126.9,119.4,114.7,113.7,108.3,107.3,106.7,106.1,101.2$, 86.0, 55.7, 52.6, 21.6; HR-ESI-MS $(m / z)$ : calcd. for $\mathrm{C}_{26} \mathrm{H}_{22} \mathrm{NO}_{7} \mathrm{~S}[\mathrm{M}+\mathrm{H}]^{+}, 492.1111$, found 492.1109 . 
Total Synthesis of (+)-Decursivine (2).

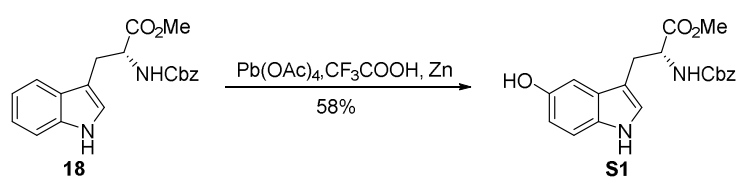

Compound S1. Compound 18 (7.04 g, $20 \mathrm{mmol})$ was dissolved in $\mathrm{CF}_{3} \mathrm{CO}_{2} \mathrm{H}(57 \mathrm{~mL})$ at room temperature and left to stand for $2.5 \mathrm{~h}$. The solution of $\mathrm{Pb}(\mathrm{OAc})_{4}(19.83 \mathrm{~g}$, $44.67 \mathrm{mmol})$ in dry $\mathrm{CH}_{2} \mathrm{Cl}_{2}(460 \mathrm{~mL})$ was then added at $10{ }^{\circ} \mathrm{C}$. The dark brown mixture was stirred for $2 \mathrm{~h}$ and zinc powder $(15.0 \mathrm{~g})$ was added to the solution. After $2 \mathrm{~h}$, water $(200 \mathrm{~mL})$ was added and the aqueous solution was extracted with $\mathrm{CH}_{2} \mathrm{Cl}_{2}$. The combined extracts were washed with $\mathrm{H}_{2} \mathrm{O}$ and dried. After the removal of the solvent under reduced pressure, the crude product was purified by column chromatography on silica gel $(\mathrm{DCM} / \mathrm{MeOH}=30: 1)$ to give the pure $\mathbf{S 1}(4.27 \mathrm{~g}, 11.6$ mmol, $58 \%$ yield $)$ as a colorless oil. $[a]_{D}^{20}=+18.0(\mathrm{c}=0.34, \mathrm{MeOH}) ;{ }^{1} \mathrm{H}$ NMR $(400$ $\left.\mathrm{MHz}, \mathrm{CD}_{3} \mathrm{OD}\right): \delta 7.28(\mathrm{~m}, 5 \mathrm{H}), 7.16(\mathrm{~d}, J=8.6,1 \mathrm{H}), 6.99(\mathrm{~s}, 1 \mathrm{H}), 6.92(\mathrm{~s}, 1 \mathrm{H}), 6.68$ $(\mathrm{d}, J=8.5,1 \mathrm{H}), 5.02(\mathrm{~s}, 2 \mathrm{H}), 4.59-4.42(\mathrm{~m}, 1 \mathrm{H}), 3.64(\mathrm{~s}, 3 \mathrm{H}), 3.20(\mathrm{dd}, J=14.6,5.7$, $1 \mathrm{H}), 3.07(\mathrm{dd}, J=14.6,7.9,1 \mathrm{H}) ;{ }^{13} \mathrm{C} \mathrm{NMR}\left(100 \mathrm{MHz}, \mathrm{CD}_{3} \mathrm{OD}\right): \delta 173.1,157.0$, 150.0, 136.7, 131.6, 128.0, 127.5, 127.3, 123.9, 111.4, 111.1, 108.5, 102.0, 66.2, 55.0, 51.3, 27.4; HR-ESI-MS (m/z): calcd. for $\mathrm{C}_{20} \mathrm{H}_{20} \mathrm{~N}_{2} \mathrm{O}_{5} \mathrm{Na}[\mathrm{M}+\mathrm{Na}]^{+}, 391.1264$, found 391.1265 .

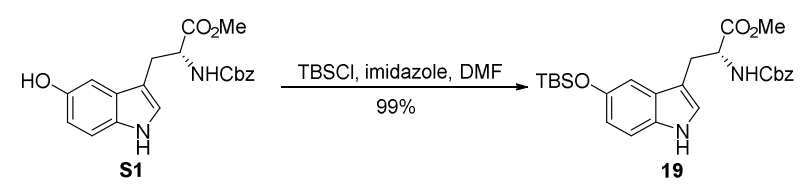

Compound 19. To a stirred solution of compound S1 (4.05 g, $11 \mathrm{mmol})$ and imidazole $(1.65 \mathrm{~g}, 24.2 \mathrm{mmol})$ in dry DMF $(30 \mathrm{~mL})$ was added tert-butyldimethylchlorosilane $(1.99 \mathrm{~g}, 13.2 \mathrm{mmol})$. The mixture was stirred $2 \mathrm{~h}$. After the removal of the solvent under reduced pressure, water $(50 \mathrm{~mL})$ was added and the aqueous solution was extracted with ethyl acetate $(3 \times 50 \mathrm{~mL})$. The combined organic phase was dried over anhydrous $\mathrm{Na}_{2} \mathrm{SO}_{4}$. The solution was concentrated in vacuo, and the crude product was purified by column chromatography on silica gel (petroleum ether/acetone $=5: 1)$ to give $19(5.25 \mathrm{~g}, 10.9 \mathrm{mmol}, 58 \%$ yield) as a colorless oil. $[a]_{D}^{20}=-21.8\left(\mathrm{c}=0.65, \mathrm{CHCl}_{3}\right) ;{ }^{1} \mathrm{H} \mathrm{NMR}\left(400 \mathrm{MHz}, \mathrm{CDCl}_{3}\right): \delta 7.92(\mathrm{~s}$, $1 \mathrm{H}), 7.18-7.08(\mathrm{~m}, 5 \mathrm{H}), 6.96(\mathrm{~d}, J=8.7,1 \mathrm{H}), 6.77(\mathrm{~s}, 1 \mathrm{H}), 6.68(\mathrm{~s}, 1 \mathrm{H}), 6.56(\mathrm{dd}, J=$ $8.6,1.9,1 \mathrm{H}), 5.15(\mathrm{~d}, J=7.9,1 \mathrm{H}), 4.90(\mathrm{q}, J=12.0,2 \mathrm{H}), 4.50(\mathrm{dd}, J=13.0,5.3,1 \mathrm{H})$, $3.49(\mathrm{~s}, 3 \mathrm{H}), 3.05(\mathrm{~d}, J=5.2,2 \mathrm{H}), 0.83(\mathrm{~s}, 9 \mathrm{H}),-0.00(\mathrm{~s}, 6 \mathrm{H}) ;{ }^{13} \mathrm{C}$ NMR $(100 \mathrm{MHz}$, $\left.\mathrm{CDCl}_{3}\right): \delta 172.5,156.0,149.4,136.3,131.7,128.5,128.3,128.2,123.7,116.5,111.6$, 109.2, 108.0, 67.0, 54,5, 52.4, 27.8, 25.8, 18.2, -4.3, -4.4; HR-ESI-MS (m/z): calcd. for $\mathrm{C}_{26} \mathrm{H}_{34} \mathrm{~N}_{2} \mathrm{O}_{5} \mathrm{SiNa}[\mathrm{M}+\mathrm{Na}]^{+}, 505.2129$, found 505.2133. 


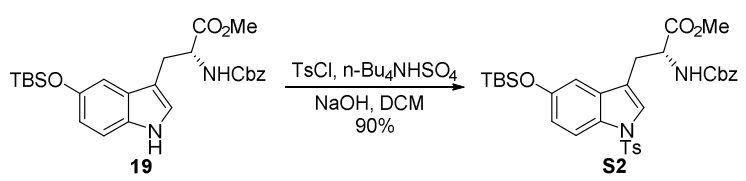

Compound S2. A solution of compound 19 (2.41 g, $5 \mathrm{mmol})$ in anhydrous DCM (60 $\mathrm{mL})$ was treated with freshly pulverized (important for reproducibility) $\mathrm{NaOH}(0.60 \mathrm{~g}$, $15 \mathrm{mmol})$ and $\mathrm{n}-\mathrm{Bu}_{4} \mathrm{NHSO}_{4}(1.70 \mathrm{~g}, 5 \mathrm{mmol})$. TsCl $(1.91 \mathrm{~g}, 10 \mathrm{mmol})$ was added and the reaction was vigorously stirred for $3 \mathrm{~h}$. The crude reaction mixture was washed with saturated aqueous $\mathrm{NaHCO}_{3}(50 \mathrm{~mL})$ and the aqueous phase back-extracted with $\mathrm{CH}_{2} \mathrm{Cl}_{2}(80 \mathrm{~mL})$. The combined organic phases were dried over $\mathrm{Na}_{2} \mathrm{SO}_{4}$, concentrated, and purified by column chromatography on silica gel (petroleum ether/acetone $=10$ : 1) to provide $\mathbf{S 2}(2.86 \mathrm{~g}, 4.5 \mathrm{mmol}, 90 \%)$ as a colorless oil. $[a]_{D}^{20}=-25.2(\mathrm{c}=0.65$, $\left.\mathrm{CHCl}_{3}\right) ;{ }^{1} \mathrm{H} \mathrm{NMR}\left(400 \mathrm{MHz}, \mathrm{CDCl}_{3}\right): \delta 7.62(\mathrm{~d}, J=8.8,1 \mathrm{H}), 7.50(\mathrm{~d}, J=8.1,2 \mathrm{H})$, $7.26-7.10(\mathrm{~m}, 6 \mathrm{H}), 6.99(\mathrm{~d}, J=7.9,2 \mathrm{H}), 6.67-6.63(\mathrm{~m}, 2 \mathrm{H}), 5.08(\mathrm{~d}, J=7.8,1 \mathrm{H})$, 5.00-4.89 (m, 2H), $4.51(\mathrm{dd}, J=12.8,5.4,1 \mathrm{H}), 3.48(\mathrm{~s}, 3 \mathrm{H}), 2.98(\mathrm{~m}, 2 \mathrm{H}), 2.14(\mathrm{~s}$, $3 \mathrm{H}), 0.80(\mathrm{~s}, 9 \mathrm{H}),-0.00(\mathrm{~s}, 6 \mathrm{H}) ;{ }^{13} \mathrm{C} \mathrm{NMR}\left(100 \mathrm{MHz}, \mathrm{CDCl}_{3}\right): \delta 171.7,155.7,152.2$, $144.8,136.1,135.1,132.0,130.2,129.8,128.6,128.3,128.2,126.7,125.3,118.3$, 116.9, 114.5, 109.2, 67.2, 53.8, 52.5, 27.8, 25.7, 21.5, 18.1, -4.4; HR-ESI-MS $(\mathrm{m} / \mathrm{z})$ : calcd. for $\mathrm{C}_{33} \mathrm{H}_{40} \mathrm{~N}_{2} \mathrm{O}_{7} \mathrm{SSiNa}[\mathrm{M}+\mathrm{Na}]^{+}, 659.2218$, found 659.2215 .

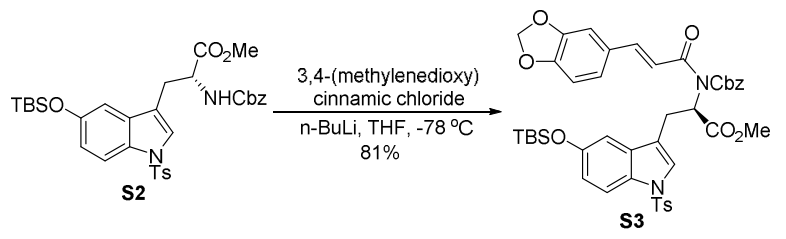

Compound S3. To a solution of $\mathbf{S 2}(1.97 \mathrm{~g}, 3.1 \mathrm{mmol})$ in dry THF $(13 \mathrm{~mL})$ was added dropwise a solution of $\mathrm{nBuLi}\left(1.24 \mathrm{~mL}, 3.1 \mathrm{mmol}, 2.5 \mathrm{M}\right.$ in hexane) at $-78^{\circ} \mathrm{C}$ under inert atmosphere. The reaction was stirred for $30 \mathrm{~min}$ at this temperature. Then a solution of 3,4-(methylenedioxy) cinnamic chloride $(0.65 \mathrm{~g}, 3.1 \mathrm{mmol})$ in dry THF $(3 \mathrm{~mL})$ was added dropwise and the reaction mixture was stirred for $3 \mathrm{~h}$ at $-78^{\circ} \mathrm{C}$. The reaction was quenched with saturated aqueous $\mathrm{NH}_{4} \mathrm{Cl}$ and the aqueous layer was extracted with $\mathrm{Et}_{2} \mathrm{O}(3 \times 10 \mathrm{~mL})$. The combined organic phases were washed with saturated aqueous $\mathrm{NaCl}$, dried over $\mathrm{MgSO}_{4}$, and concentrated under reduced pressure. The crude product was purified by column chromatography on silica gel (petroleum ether/acetone $=5: 1)$ to afford $\mathbf{S 3}(2.03 \mathrm{~g}, 2.51 \mathrm{mmol}, 81 \%)$ as a colorless oil. $[a]_{D}^{20}=+68.1\left(\mathrm{c}=0.37, \mathrm{CHCl}_{3}\right) ;{ }^{1} \mathrm{H} \operatorname{NMR}\left(400 \mathrm{MHz}, \mathrm{CDCl}_{3}\right): \delta 7.59(\mathrm{~d}, J=8.9,1 \mathrm{H})$, $7.48(\mathrm{~d}, J=8.2,2 \mathrm{H}), 7.36(\mathrm{~d}, J=15.5,1 \mathrm{H}), 7.21-7.15(\mathrm{~m}, 3 \mathrm{H}), 7.13(\mathrm{~s}, 1 \mathrm{H})$, 7.09-7.02 (m, 2H), $6.94(\mathrm{~d}, J=8.1,2 \mathrm{H}), 6.84(\mathrm{~d}, J=15.5,1 \mathrm{H}), 6.77-6.68(\mathrm{~m}, 3 \mathrm{H})$, $6.69-6.61(\mathrm{~m}, 2 \mathrm{H}), 5.85(\mathrm{~s}, 2 \mathrm{H}), 5.31(\mathrm{dd}, J=9.6,5.0,1 \mathrm{H}), 4.90(\mathrm{~d}, J=11.9,1 \mathrm{H})$, $4.78(\mathrm{~d}, J=11.9,1 \mathrm{H}), 3.48(\mathrm{~s}, 3 \mathrm{H}), 3.33(\mathrm{dd}, J=15.3,5.0,1 \mathrm{H}), 3.14(\mathrm{dd}, J=15.2$, 9.8, 1H), $2.08(\mathrm{~s}, 3 \mathrm{H}), 0.81(\mathrm{~s}, 9 \mathrm{H}),-0.00(\mathrm{~s}, 6 \mathrm{H}) ;{ }^{13} \mathrm{C} \mathrm{NMR}\left(100 \mathrm{MHz}, \mathrm{CDCl}_{3}\right): \delta$ 
$170.5,167.9,153.6,152.0,149.7,148.3,144.8,144.5,135.2,134.3,131.9,130.1$, $129.7,129.2,128.9,128.8,128.6,126.7,125.2,124.8,118.3,118.2,118.1,114.2$, 109.3, 108.5, 106.8, 101.5, 69.1, 56.7, 52.5, 25.7, 25.1, 21.5, 18.1, -4.4; HR-ESI-MS $(\mathrm{m} / \mathrm{z})$ : calcd. for $\mathrm{C}_{43} \mathrm{H}_{47} \mathrm{~N}_{2} \mathrm{O}_{10} \mathrm{SiS}[\mathrm{M}+\mathrm{H}]^{+}, 811.2721$, found 811.2726.

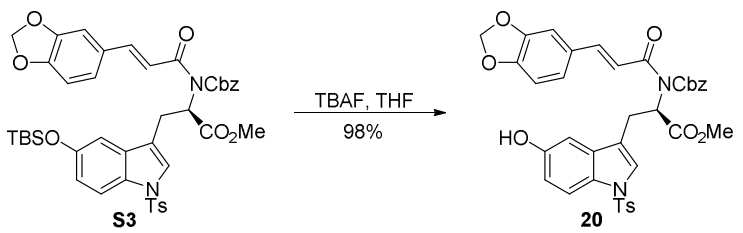

Compound 20. TBAF $3 \mathrm{H}_{2} \mathrm{O}(1.21 \mathrm{~g}, 3.85 \mathrm{mmol})$ was added to a solution of compound S3 (2.84 g, $3.50 \mathrm{mmol})$ in THF $(20 \mathrm{~mL})$. The solution was stirred at room temperature for $1 \mathrm{~h}$. Saturated aqueous $\mathrm{NH}_{4} \mathrm{Cl}(30 \mathrm{~mL})$ was added and extracted with ethyl acetate $(3 \times 50 \mathrm{~mL})$. The combined organic phase was dried over anhydrous $\mathrm{Na}_{2} \mathrm{SO}_{4}$. After the removal of solvent under reduced pressure, the crude product was purified by column chromatography on silica gel (petroleum ether/acetone $=3: 1$ ) to give the compound $20(2.37 \mathrm{~g}, 3.40 \mathrm{mmol}, 98 \%)$ as a white solid mp.88 - $91{ }^{\circ} \mathrm{C}$. $[a]_{D}^{20}=+106.6\left(\mathrm{c}=0.59, \mathrm{CHCl}_{3}\right) ;{ }^{1} \mathrm{H} \mathrm{NMR}\left(400 \mathrm{MHz}, \mathrm{CDCl}_{3}\right): \delta 7.63(\mathrm{~d}, J=8.8$, $1 \mathrm{H}), 7.53(\mathrm{~d}, J=8.2,2 \mathrm{H}), 7.41(\mathrm{~d}, J=15.5,1 \mathrm{H}), 7.29-7.22(\mathrm{~m}, 3 \mathrm{H}), 7.20(\mathrm{~s}, 1 \mathrm{H})$, $7.16-7.10(\mathrm{~m}, 2 \mathrm{H}), 7.00(\mathrm{~d}, J=8.2,2 \mathrm{H}), 6.91(\mathrm{~d}, J=15.5,1 \mathrm{H}), 6.80-6.73(\mathrm{~m}, 3 \mathrm{H})$, $6.72-6.60(\mathrm{~m}, 2 \mathrm{H}), 5.91(\mathrm{~s}, 2 \mathrm{H}), 5.41(\mathrm{dd}, J=9.3,5.4,1 \mathrm{H}), 5.00(\mathrm{~d}, J=11.8,1 \mathrm{H})$, $4.88(\mathrm{~d}, J=11.8,1 \mathrm{H}), 3.53(\mathrm{~s}, 3 \mathrm{H}), 3.40(\mathrm{dd}, J=15.1,5.3,1 \mathrm{H}), 3.17(\mathrm{dd}, J=15.1$, 9.5, 1H), $2.12(\mathrm{~s}, 3 \mathrm{H}) ;{ }^{13} \mathrm{C}$ NMR $\left(100 \mathrm{MHz}, \mathrm{CDCl}_{3}\right): \delta 170.4,168.2,153.6,152.5$, $149.8,148.3,145.0,144.6,135.1,134.3,131.9,129.8,129.6,129.1,129.0,128.9$, $128.8,126.7,125.6,124.9,118.1,118.0,114.6,113.9,108.5,106.7,104.5,101.6$, 69.2, 56.6, 52.5, 25.3, 21.4; HR-ESI-MS $(m / z)$ : calcd. for $\mathrm{C}_{37} \mathrm{H}_{32} \mathrm{~N}_{2} \mathrm{O}_{10} \mathrm{SNa}[\mathrm{M}+\mathrm{Na}]^{+}$, 719.1670 , found 719.1668 .

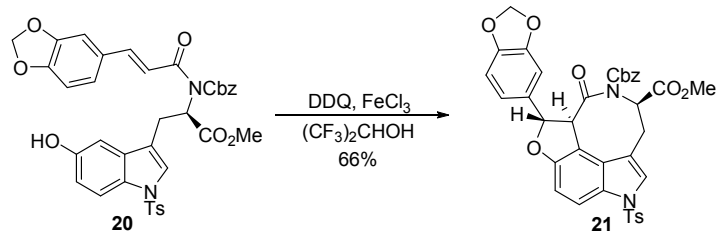

Compound 21. To an oven dried Schlenk tube equipped with a stir-bar, compound 20 (35 mg, $0.05 \mathrm{mmol}$ ), DDQ (23 mg, $0.10 \mathrm{mmol}$ ), $\mathrm{FeCl}_{3}(4 \mathrm{mg}, 0.025 \mathrm{mmol}$, in the glovebox) were added. Then the reaction tube was allowed to be vacuumed and purged with nitrogen for three times. HFIP $(5 \mathrm{~mL})$ was added under nitrogen. The Schlenk tube was allowed to stir at room temperature for $2.5 \mathrm{~h}$. The mixture was quenched with water and extracted with EtOAc $(15 \mathrm{~mL} \times 3)$. The combined organic layers were washed with brine, dried over anhydrous $\mathrm{Na}_{2} \mathrm{SO}_{4}$ and concentrated. The crude product was subjected to flash chromatography on silica gel (DCM/acetone $=$ $100: 1)$ to afford $21(23 \mathrm{mg}, 0.033 \mathrm{mmol}, 66 \%)$ as a colorless oil. $[a]_{D}^{20}=+85.2(\mathrm{c}=$ $\left.0.36, \mathrm{CHCl}_{3}\right) ;{ }^{1} \mathrm{H}$ NMR $\left(400 \mathrm{MHz}, \mathrm{CDCl}_{3}\right): \delta 7.85(\mathrm{~d}, J=8.9,1 \mathrm{H}), 7.64(\mathrm{~d}, J=8.2$, $2 \mathrm{H}), 7.37(\mathrm{~s}, 1 \mathrm{H}), 7.31-7.21(\mathrm{~m}, 5 \mathrm{H}), 7.17(\mathrm{~d}, J=8.2,2 \mathrm{H}), 6.90-6.78(\mathrm{~m}, 3 \mathrm{H}), 6.71(\mathrm{~d}$, 
$J=7.9,1 \mathrm{H}), 6.28(\mathrm{~d}, J=7.9,1 \mathrm{H}), 5.86(\mathrm{~s}, 2 \mathrm{H}), 5.20-5.11(\mathrm{~m}, 2 \mathrm{H}), 5.10-5.01(\mathrm{~m}, 1 \mathrm{H})$, $4.61(\mathrm{~d}, J=8.1,1 \mathrm{H}), 3.68-3.56(\mathrm{~m}, 1 \mathrm{H}), 3.56-3.39(\mathrm{~m}, 4 \mathrm{H}), 2.27(\mathrm{~s}, 3 \mathrm{H}) ;{ }^{13} \mathrm{C} \mathrm{NMR}$ $\left(100 \mathrm{MHz}, \mathrm{CDCl}_{3}\right): \delta 169.1,168.7,156.2,152.8,148.1,147.8,145.2,134.9,134.6$, $134.5,130.77,130.0,128.6,128.6,128.2,126.9,126.4,126.3,119.9,116.0,115.3$, $112.0,108.5,107.9,106.4,101.2,84.6,69.1,58.4,57.4,52.9,27.0,21.6$; HR-ESI-MS $(m / z)$ : calcd. for $\mathrm{C}_{37} \mathrm{H}_{30} \mathrm{~N}_{2} \mathrm{O}_{10} \mathrm{SNa}[\mathrm{M}+\mathrm{Na}]^{+}, 717.1513$, found 717.1527.

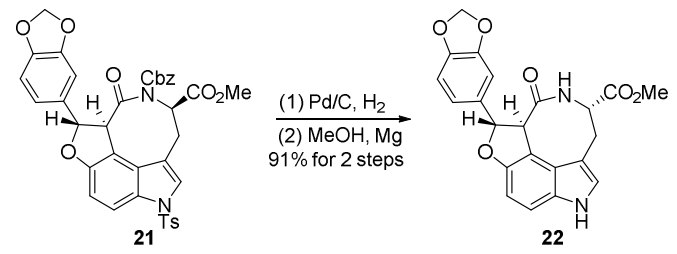

Compound 22. To a solution of 21 (37 mg, $0.053 \mathrm{mmol})$ in a THF/MeOH (2:1, $3 \mathrm{~mL})$ was added $10 \% \mathrm{Pd} / \mathrm{C}(20 \mathrm{mg})$ and the vessel purged several times with $\mathrm{H}_{2}$. The mixture was stirred under $1 \mathrm{~atm}$ of $\mathrm{H}_{2}$ for $0.5 \mathrm{~h}$ and then filtered over a pad of Celite. After washing the solids with a $1: 1 \mathrm{CH}_{2} \mathrm{Cl}_{2} / \mathrm{MeOH}$ mixture $(15 \mathrm{~mL})$, the collected filtrates were concentrated in vacuo. Without any further purification, $\mathrm{Mg}$ turnings (38 $\mathrm{mg}, 1.59 \mathrm{mmol}$ ) was added to the methanolic solution $(2 \mathrm{~mL})$ of the crude product. The reaction mixture was stirred at $23{ }^{\circ} \mathrm{C}$ for $30 \mathrm{~min}$. After completion of the reaction, it was quenched with saturated aqueous $\mathrm{NH}_{4} \mathrm{Cl}(10 \mathrm{~mL})$ and extracted with ethyl acetate $(3 \times 10 \mathrm{~mL})$, washed with brine $(2 \times 10 \mathrm{~mL})$, dried over $\mathrm{Na}_{2} \mathrm{SO}_{4}$. After removal of the solvent, the crude residue was purified with column chromatography on silica gel $(\mathrm{DCM} /$ acetone $=30: 1)$ to yield $22(20 \mathrm{mg}, 0.048 \mathrm{mmol}, 91 \%$ for 2 steps $)$ as a colorless oil. $[a]_{D}^{20}=+96.1\left(\mathrm{c}=0.30, \mathrm{CHCl}_{3}\right) ;{ }^{1} \mathrm{H}$ NMR $\left(400 \mathrm{MHz}, \mathrm{CDCl}_{3}\right): \delta$ $8.19(\mathrm{~s}, 1 \mathrm{H}), 7.24(\mathrm{~d}, J=8.8,1 \mathrm{H}), 7.06(\mathrm{~s}, 1 \mathrm{H}), 7.06-6.99(\mathrm{~m}, 2 \mathrm{H}), 6.85(\mathrm{~d}, J=8.7$, $1 \mathrm{H}), 6.81(\mathrm{~d}, J=8.5,1 \mathrm{H}), 6.44-6.37(\mathrm{~m}, 2 \mathrm{H}), 5.95(\mathrm{~s}, 2 \mathrm{H}), 4.94(\mathrm{t}, J=10.3,1 \mathrm{H}), 4.51$ $(\mathrm{d}, J=9.5,1 \mathrm{H}), 3.82(\mathrm{~s}, 3 \mathrm{H}), 3.50(\mathrm{~d}, J=16.3,1 \mathrm{H}), 3.17(\mathrm{dd}, J=16.1,11.6,1 \mathrm{H}) ;{ }^{13} \mathrm{C}$ NMR $\left(100 \mathrm{MHz}, \mathrm{CDCl}_{3}\right): \delta 171.5,169.7,153.5,148.0,147.5,135.3,132.5,124.4$, 122.2 , 119.9, 112.8, 111.9, 109.8, 108.3, 106.7, 106.0, 101.1, 84.3, 55.6, 54.5, 53.0, 34.2; HR-ESI-MS $(m / z)$ : calcd. for $\mathrm{C}_{22} \mathrm{H}_{18} \mathrm{~N}_{2} \mathrm{O}_{6} \mathrm{Na}[\mathrm{M}+\mathrm{Na}]^{+}$, 429.1057, found 429.1051 .

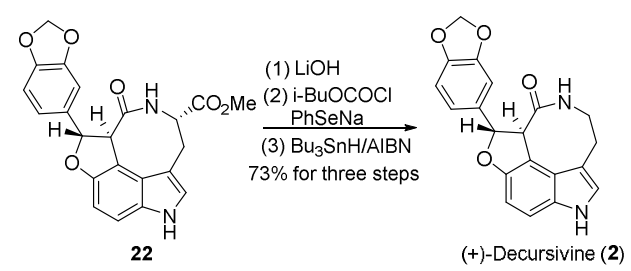

(+)-Decursivine (2). To the solution of compound $22(65 \mathrm{mg}, 0.16 \mathrm{mmol})$ in $10 \mathrm{~mL}$ $\mathrm{THF} / \mathrm{H}_{2} \mathrm{O}(4 / 1,5 \mathrm{~mL})$ was added $\mathrm{LiOH} \cdot \mathrm{H}_{2} \mathrm{O}(0.47 \mathrm{~g}, 1.12 \mathrm{mmol})$ and the mixture was stirred for $6 \mathrm{~h}$. The reaction was then quenched by careful addition of aqueous 1 $\mathrm{N} \mathrm{HCl}$ until the $\mathrm{pH}$ of the solution was approximately 6.5. The aqueous solution was saturated with solid $\mathrm{NaCl}$. The aqueous phase was extracted with EtOAc $(3 \times 20 \mathrm{~mL})$. 
The combined organic phases were washed with saturated aqueous $\mathrm{NaCl}$, dried over $\mathrm{Na}_{2} \mathrm{SO}_{4}$, and then concentrated. The product acid obtained as a white solid was then directly used without further purification. The acid thus prepared was then added into the THF $(3 \mathrm{~mL})$ solution of isobutyl chloroformate $(39 \mu \mathrm{L}, 0.30 \mathrm{mmol})$ and $N$-methylmorpholine $(33 \mu \mathrm{L}, 0.30 \mathrm{mmol})$ at $-10{ }^{\circ} \mathrm{C}$. The solution was stirred at $-10{ }^{\circ} \mathrm{C}$ for $15 \mathrm{~min}$ and at $\mathrm{rt}$ for another $15 \mathrm{~min}$. The solution was again cooled to $-10^{\circ} \mathrm{C}$. The THF solution of sodium phenylselenide, which was prepared by reaction of benzeneselenol $(33 \mu \mathrm{L}, 0.30 \mathrm{mmol})$ and sodium hydride $(8 \mathrm{mg}, 0.33 \mathrm{mmol})$ in anhydrous THF $(3 \mathrm{~mL})$ at $0{ }^{\circ} \mathrm{C}$, was then added through a cannula. The resulting mixture was stirred at $-10{ }^{\circ} \mathrm{C}$ for $20 \mathrm{~min}$ and then at $\mathrm{rt}$ for another $2 \mathrm{~h}$. The volatiles were removed under reduced pressure, the residue was dissolved in anhydrous benzene $(5 \mathrm{~mL}) . \mathrm{Bu}_{3} \mathrm{SnH}(150 \mu \mathrm{L}, 0.60 \mathrm{mmol})$ and AIBN $(16 \mathrm{mg}, 0.10 \mathrm{mmol})$ were added, and the mixture was refluxed for $8 \mathrm{~h}$. The solvent was then removed under reduced pressure, and the residue was purified by column chromatography on silica gel (petroleum ether/acetone $=2: 1)$ to give $(+)$-decursivine (2) $(41 \mathrm{mg}, 0.12 \mathrm{mmol}$, $73 \%$ for 3 steps) as a white solid mp.251 - $253{ }^{\circ} \mathrm{C}^{[3]}[a]_{D}^{20}=+262.2(\mathrm{c}=0.02$, $\mathrm{MeOH}) ;{ }^{1} \mathrm{H}$ NMR (400 MHz, pyridine- $\left.d_{5}\right): \delta 12.04(\mathrm{~s}, 1 \mathrm{H}), 8.82(\mathrm{dd}, J=9.6,4.2,1 \mathrm{H})$, $7.46(\mathrm{~d}, J=8.6,1 \mathrm{H}), 7.36(\mathrm{~s}, 1 \mathrm{H}), 7.30-7.26(\mathrm{~m}, 2 \mathrm{H}), 7.10-7.00(\mathrm{~m}, 2 \mathrm{H}), 6.91(\mathrm{~d}, J=$ $7.9,1 \mathrm{H}), 5.95(\mathrm{~d}, J=2.9,2 \mathrm{H}), 5.03(\mathrm{~d}, J=9.6,1 \mathrm{H}), 4.23-4.14(\mathrm{~m}, 1 \mathrm{H}), 3.63-3.55(\mathrm{~m}$, $1 \mathrm{H}), 3.23-3.16(\mathrm{~m}, 2 \mathrm{H}) ;{ }^{13} \mathrm{C}$ NMR $\left(150 \mathrm{MHz}\right.$, pyridine- $\left.d_{5}\right): \delta 171.5,153.3,148.3$, $147.6,136.9,134.0,125.7,123.6,120.1,114.6,112.1,111.7,108.5,107.2,105.1$, 101.5, 84.9, 55.5, 41.1, 30.4; HR-ESI-MS $(\mathrm{m} / z)$ : calcd. for $\mathrm{C}_{20} \mathrm{H}_{16} \mathrm{~N}_{2} \mathrm{O}_{4} \mathrm{Na}[\mathrm{M}+\mathrm{Na}]^{+}$, 371.1002 , found 371.1000 .

\section{Total synthesis of $4,4^{\prime \prime}$-bis(N-feruloyl)serotonin (4).}

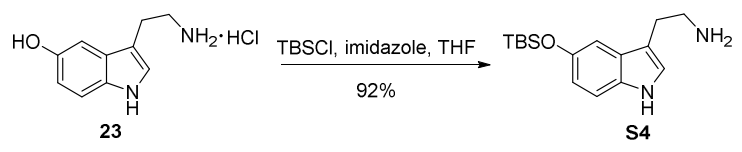

Compound S4. 5-Hydroxytryptamine hydrochloride 23 (1.06 g, $5.00 \mathrm{mmol}$ ) was dissolved in $25 \mathrm{~mL}$ of THF, followed by the addition of imidazole (1.12 g, 16.50 mmol) and tert-butyldimethylsilyl chloride (TBSCl) (1.66 g, $11.00 \mathrm{mmol})$. The reaction was stirred overnight at room temperature under $\mathrm{N}_{2}$ atmosphere in which a precipitate formed. The precipitate was then filtered and washed with dichloromethane (DCM). The solid was dissolved in $50.0 \mathrm{~mL}$ of $0.5 \mathrm{M} \mathrm{NaOH}$ and extracted with ether $(3 \times 50 \mathrm{~mL})$. The organic phase then was washed with deionized water $(2 \times 50 \mathrm{~mL})$ and then dried over $\mathrm{MgSO}_{4}$. Column chromatography on silica gel $(\mathrm{MeOH} / \mathrm{DCM}=1: 5)$ afforded $\mathbf{S 4}(1.34 \mathrm{~g}, 4.60 \mathrm{mmol}, 92 \%)$ a colorless oil. ${ }^{[1]}{ }^{1} \mathrm{H}$ NMR (400 MHz, $\left.\mathrm{CD}_{3} \mathrm{OD}\right): \delta 7.02(\mathrm{~d}, J=8.6,1 \mathrm{H}), 6.82(\mathrm{~s}, 1 \mathrm{H}), 6.81(\mathrm{~s}, 1 \mathrm{H}), 6.51(\mathrm{dd}$, $J=8.6,1.6,1 \mathrm{H}), 4.62(\mathrm{~s}, 2 \mathrm{H}), 2.68(\mathrm{~m}, 2 \mathrm{H}), 2.63(\mathrm{~m}, 2 \mathrm{H}), 0.83(\mathrm{~s}, 9 \mathrm{H}), 0.00(\mathrm{~s}, 6 \mathrm{H})$;

${ }^{13} \mathrm{C}$ NMR $\left(100 \mathrm{MHz}, \mathrm{CD}_{3} \mathrm{OD}\right): \delta 148.3,132.7,127.9,123.3,115.2,111.5,111.3$, 107.6, 41.6, 28.2, 25.1, 17.8, -5.4; HR-ESI-MS $(\mathrm{m} / \mathrm{z})$ : calcd. for $\mathrm{C}_{16} \mathrm{H}_{27} \mathrm{~N}_{2} \mathrm{OSi}[\mathrm{M}+$ $\mathrm{H}]^{+}, 291.1887$, found 291.1894 . 


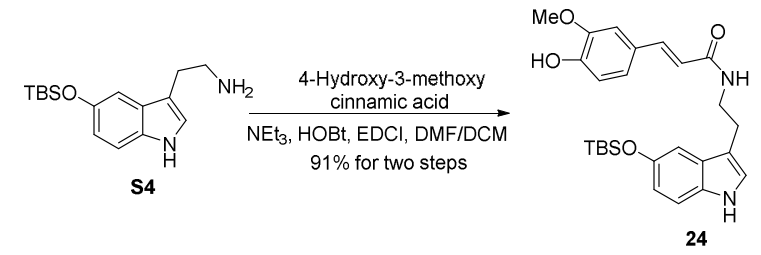

Compound 24. Triethylamine $(0.19 \mathrm{ml}, 1.36 \mathrm{mmol})$, 1-hydroxybenzotriazole (184 $\mathrm{mg}, 1.36 \mathrm{mmol})$ and EDCI (261 mg, $1.36 \mathrm{mmol})$ were added to a solution S4 (394 mg, $1.36 \mathrm{mmol})$ and ferulic acid (264 mg, $1.36 \mathrm{mmol})$ in DMF $(1.5 \mathrm{ml})$ and DCM (10 ml). After the mixture was stirred over night at room temperature under nitrogen, it was concentrated in vacuo. The residue was treated with water $(15 \mathrm{ml})$ and extracted with ethyl acetate $(3 \times 10 \mathrm{ml})$. The organic extract was washed with brine $(50 \mathrm{ml})$ and dried over $\mathrm{Na}_{2} \mathrm{SO}_{4}$. Column chromatography (petroleum ether/acetone $=2: 1$ ) afforded 24 (577 mg, $1.24 \mathrm{mmol}, 91 \%$ ) as a colorless oil. ${ }^{1} \mathrm{H} \mathrm{NMR}\left(400 \mathrm{MHz}, \mathrm{CDCl}_{3}\right): \delta 7.91(\mathrm{~s}$, $1 \mathrm{H}), 7.33(\mathrm{~d}, J=15.5,1 \mathrm{H}), 7.03(\mathrm{~d}, J=8.6,1 \mathrm{H}), 6.83-6,78(\mathrm{~m}, 3 \mathrm{H}), 6.75(\mathrm{~s}, 1 \mathrm{H})$, $6.69(\mathrm{~d}, J=8.2,1 \mathrm{H}), 6.58(\mathrm{dd}, J=8.6,1.8,1 \mathrm{H}), 5.97(\mathrm{~d}, J=15.5,1 \mathrm{H}), 5.54(\mathrm{~s}, 1 \mathrm{H})$, $3.68(\mathrm{~s}, 3 \mathrm{H}), 3.53(\mathrm{dd}, J=12.1,6.1,2 \mathrm{H}), 2.79(\mathrm{t}, J=6.4,2 \mathrm{H}), 0.81(\mathrm{~s}, 9 \mathrm{H}),-0.00(\mathrm{~s}$, $6 \mathrm{H}) ;{ }^{13} \mathrm{C} \mathrm{NMR}\left(100 \mathrm{MHz}, \mathrm{CDCl}_{3}\right): \delta 166.3,149.3,147.4,146.7,140.9,132.0,128.1$, 127.4, 123.0, 122.1, 118.3, 116.4, 114.7, 112.5, 111.6, 109.6, 108.2, 55.9, 39.8, 25.8, 25.3, 18.2, -4.4; HR-ESI-MS (m/z): calcd. for $\mathrm{C}_{26} \mathrm{H}_{35} \mathrm{~N}_{2} \mathrm{O}_{4} \mathrm{Si}[\mathrm{M}+\mathrm{H}]^{+}, 467.2361$, found 467.2361 .

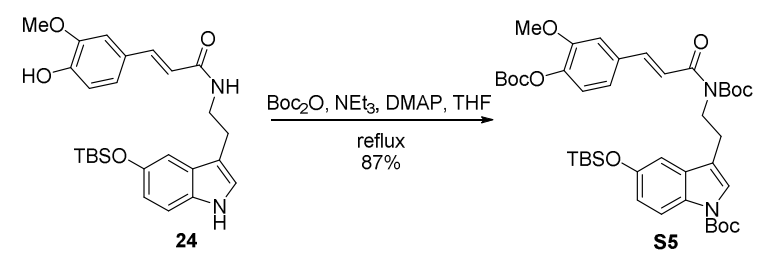

Compound S5. To a solution of compound 24 (577 mg, $1.24 \mathrm{mmol})$ in dry THF (6 $\mathrm{mL})$ was added DMAP (151 mg, $1.24 \mathrm{mmol}), \mathrm{Boc}_{2} \mathrm{O}(2.706 \mathrm{~g}, 12.4 \mathrm{mmol})$ and triethylamine $(0.86 \mathrm{~mL}, 6.2 \mathrm{mmol})$. The mixture was stirred at reflux for $1 \mathrm{~h}$. After the removal of solvent under reduced pressure, water $(50 \mathrm{~mL})$ was added and the resulting mixture was extracted with ethyl acetate $(3 \times 50 \mathrm{~mL})$. The combined organic layer was then dried over anhydrous $\mathrm{Na}_{2} \mathrm{SO}_{4}$. The solution was concentrated in vacuo and the crude product was purified by column chromatography on silica gel (petroleum ether/acetone $=10: 1)$ to give $\mathbf{S 5}(826 \mathrm{mg}, 1.09 \mathrm{mmol}, 87 \%)$ as a colorless oil. ${ }^{1} \mathrm{H}$ NMR $\left(400 \mathrm{MHz}, \mathrm{CDCl}_{3}\right): \delta 7.73(\mathrm{~s}, 1 \mathrm{H}), 7.43(\mathrm{~d}, J=15.6,1 \mathrm{H}), 7.19$ $(\mathrm{d}, J=15.6,2 \mathrm{H}), 6.99-6.86(\mathrm{~m}, 4 \mathrm{H}), 6.62(\mathrm{dd}, J=8.8,2.2,1 \mathrm{H}), 3.85-3.73(\mathrm{~m}, 2 \mathrm{H})$, $3.67(\mathrm{~s}, 3 \mathrm{H}), 2.81-2.65(\mathrm{~m}, 2 \mathrm{H}), 1.41(\mathrm{~s}, 9 \mathrm{H}), 1.33(\mathrm{~s}, 9 \mathrm{H}), 1.26(\mathrm{~s}, 9 \mathrm{H}), 0.79(\mathrm{~s}, 9 \mathrm{H})$, $-0.00(\mathrm{~s}, 6 \mathrm{H}) \cdot ;{ }^{13} \mathrm{C} \mathrm{NMR}\left(100 \mathrm{MHz}, \mathrm{CDCl}_{3}\right): \delta 168.4,153.1,151.4,151.2,149.7$, $142.3,141.5,134.1,131.6,131.6,123.9,122.8,121.9,120.9,117.6,117.5,115.7$, 111.8, 109.3, 83.6, 83.2, 83.2, 56.0, 44.9, 28.2, 28.0, 27.6, 25.8, 24.5, 18.2, -4.4; HR-ESI-MS $(m / z)$ : calcd. for $\mathrm{C}_{41} \mathrm{H}_{59} \mathrm{~N}_{2} \mathrm{O}_{10} \mathrm{Si}[\mathrm{M}+\mathrm{H}]^{+}, 767.3939$, found 767.3936. 


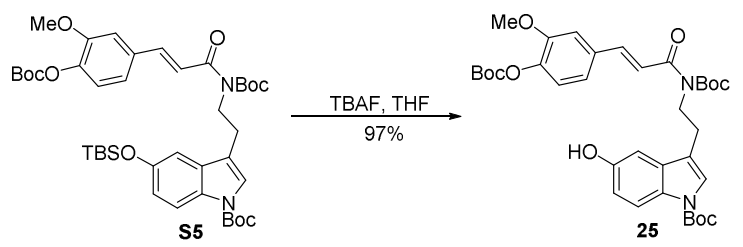

Compound 25. TBAF $3 \mathrm{H}_{2} \mathrm{O}(378 \mathrm{mg}, 1.20 \mathrm{mmol})$ was added to a solution of compound S5 (766 mg, $1.00 \mathrm{mmol})$ in THF $(5 \mathrm{~mL})$. The solution was stirred at room temperature for $1 \mathrm{~h}$. Saturated aqueous $\mathrm{NH}_{4} \mathrm{Cl}(10 \mathrm{~mL})$ was added and extracted with ethyl acetate $(3 \times 10 \mathrm{~mL})$. The combined organic phase was dried over anhydrous $\mathrm{Na}_{2} \mathrm{SO}_{4}$. After the removal of solvent under reduced pressure, the crude product was purified by column chromatography on silica gel (petroleum ether/acetone $=4: 1$ ) to give the compound $25(632 \mathrm{mg}, 0.97 \mathrm{mmol}, 97 \%)$ as a white solid mp.62 - $64{ }^{\circ} \mathrm{C} .{ }^{1} \mathrm{H}$ NMR (400 MHz, $\left.\mathrm{CDCl}_{3}\right): \delta 7.85(\mathrm{~s}, 1 \mathrm{H}), 7.55(\mathrm{~d}, J=15.6,1 \mathrm{H}), 7.32(\mathrm{~d}, J=15.6$, $1 \mathrm{H}), 7.29(\mathrm{~s}, 1 \mathrm{H}), 7.11(\mathrm{~d}, J=2.1,1 \mathrm{H}), 7.04(\mathrm{q}, J=8.2,2 \mathrm{H}), 6.99(\mathrm{~s}, 1 \mathrm{H}), 6.79$ (dd, $J$ $=8.8,2.3,1 \mathrm{H}), 3.98-3.85(\mathrm{~m}, 2 \mathrm{H}), 3.77(\mathrm{~s}, 3 \mathrm{H}), 2.91-2.73(\mathrm{~m}, 2 \mathrm{H}), 1.55(\mathrm{~s}, 9 \mathrm{H}), 1.47$ $(\mathrm{s}, 9 \mathrm{H}), 1.40(\mathrm{~s}, 9 \mathrm{H}) ;{ }^{13} \mathrm{C}$ NMR $\left(101 \mathrm{MHz}, \mathrm{CDCl}_{3}\right): \delta 168.8,153.1,152.0,151.4$, $151.2,149.7,142.6,141.6,133.9,131.6,130.1,124.0,122.8,121.8,120.9,117.3$, $116.0,113.3,111.9,104.5,83.7,83.5,83.3,56.0,45.0,28.2,28.0,27.6,24.6$; HR-ESI-MS $(m / z)$ : calcd. for $\mathrm{C}_{35} \mathrm{H}_{44} \mathrm{~N}_{2} \mathrm{O}_{10} \mathrm{Na}[\mathrm{M}+\mathrm{Na}]^{+}, 675.2888$, found 675.2889.

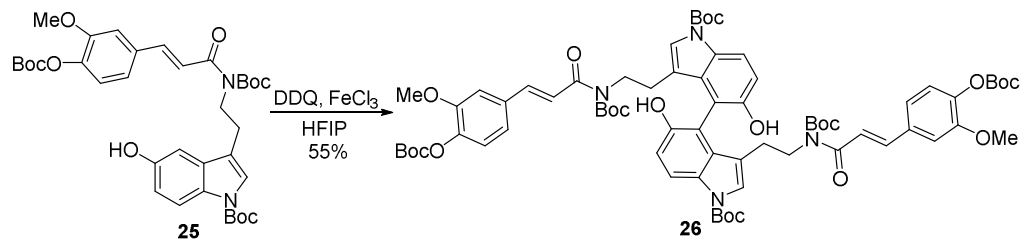

Compound 26. To an oven dried Schlenk tube equipped with a stir-bar, compound 25 (65 mg, $0.10 \mathrm{mmol}), \mathrm{FeCl}_{3}(1.6 \mathrm{mg}, 0.01 \mathrm{mmol}$, in the glovebox) were added. Then the reaction tube was allowed to be vacuumed and purged with nitrogen for three times. HFIP ( $2 \mathrm{~mL})$ and DDQ (14 $\mathrm{mg}, 0.06 \mathrm{mmol})$ were added under nitrogen. The reaction mixture was stirred at room temperature for $10 \mathrm{~min}$, at which time an additional $7 \mathrm{mg}$ of DDQ $(0.03 \mathrm{mmol})$ was added. The reaction mixture was stirred at room temperature for another $5 \mathrm{~min}$, and a third batch of $7 \mathrm{mg}$ of DDQ $(0.03 \mathrm{mmol})$ was added. The reaction mixture was allowed to stir at room temperature for another $5 \mathrm{~min}$. The mixture was quenched with water and extracted with EtOAc $(15 \mathrm{~mL} \times 3)$. The combined organic layers were washed with brine, dried over anhydrous $\mathrm{Na}_{2} \mathrm{SO}_{4}$ and concentrated. The crude product was subjected to flash chromatography on silica gel $(\mathrm{DCM} /$ acetone $=30: 1)$ to afford $26(36 \mathrm{mg}, 0.028 \mathrm{mmol}, 55 \%)$ as a colorless oil. ${ }^{1} \mathrm{H}$ NMR (400 MHz, $\left.\mathrm{CDCl}_{3}\right): \delta 8.08(\mathrm{~s}, 2 \mathrm{H}), 7.33(\mathrm{~d}, J=15.5,2 \mathrm{H}), 7.17(\mathrm{~s}, 2 \mathrm{H}), 7.09$ $(\mathrm{d}, J=15.5,2 \mathrm{H}), 7.03(\mathrm{~m}, 6 \mathrm{H}), 6.97(\mathrm{~s}, 2 \mathrm{H}), 5.47(\mathrm{~s}, 2 \mathrm{H}), 3.80(\mathrm{~s}, 6 \mathrm{H}), 3.62-3.46(\mathrm{~m}$, $2 \mathrm{H}), 3.40-3.35(\mathrm{~m}, 2 \mathrm{H}), 2.33-2.14(\mathrm{~m}, 2 \mathrm{H}), 1.96-1.89(\mathrm{~m}, 2 \mathrm{H}), 1.52(\mathrm{~s}, 18 \mathrm{H}), 1.48(\mathrm{~s}$, $18 \mathrm{H}), 1.25(\mathrm{~s}, 18 \mathrm{H}) ;{ }^{13} \mathrm{C} \mathrm{NMR}\left(100 \mathrm{MHz}, \mathrm{CDCl}_{3}\right): \delta 168.4,153.0,151.4,151.1$, $150.4,149.4,141.8,141.5,133.9,130.6,125.2,122.8,121.8,120.7,117.9,117.1$, 114.1, 111.8, 111.0, 83.7, 83.5, 83.0, 56.0, 43.8, 29.7, 28.1, 27.8, 27.6, 24.8; HR-ESI-MS $(m / z)$ : calcd. for $\mathrm{C}_{70} \mathrm{H}_{86} \mathrm{~N}_{4} \mathrm{O}_{20} \mathrm{Na}[\mathrm{M}+\mathrm{Na}]^{+}$, 1325.5733, found 
1325.5731.
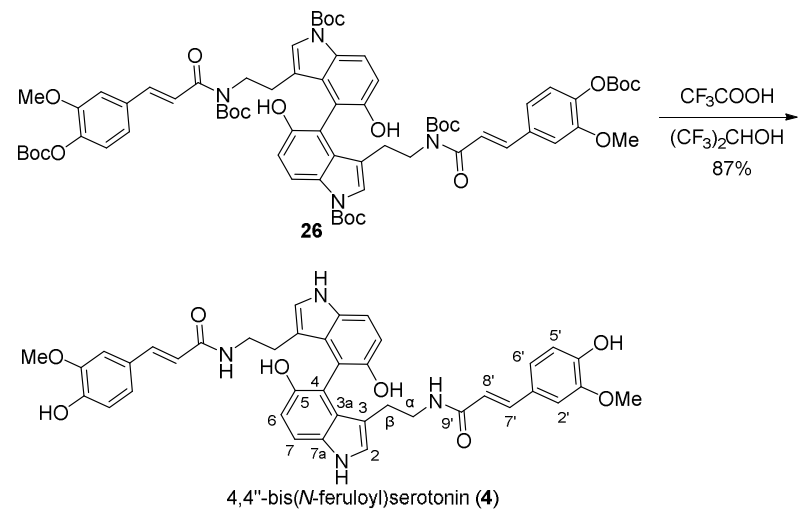

4,4"-Bis( $N$-feruloyl)serotonin (4). Trifiuoroacetic acid $(0.067 \mathrm{~mL})$ was added dropwise to a solution of $26(65 \mathrm{mg}, 0.050 \mathrm{mmol})$ in HFIP $(4 \mathrm{~mL})$ at $0^{\circ} \mathrm{C}$. The reaction mixture was stirred for $24 \mathrm{~h}$ at room temperature. The solution was concentrated by co-evaporation with toluene $(3 \times 5 \mathrm{~mL})$ and the crude product was purified by column chromatography on silica gel $(\mathrm{DCM} / \mathrm{MeOH}=12: 1)$ to give 4,4"-bis( $N$-feruloyl)serotonin (4) (31 mg, $0.043 \mathrm{mmol}, 87 \%$ ) as a white solid mp.159 - $161{ }^{\circ} \mathrm{C} .{ }^{1} \mathrm{H}$ NMR (400 MHz, DMSO- $\left.d_{6}\right): \delta 10.42$ (s, 2H), 9.38 (s, 2H), 7.75 (s, 2H), $7.37(\mathrm{t}, J=4.9,2 \mathrm{H}), 7.21(\mathrm{~d}, J=15.6,2 \mathrm{H}), 7.14(\mathrm{~d}, J=8.6,2 \mathrm{H}), 7.02(\mathrm{~d}, J=1.8,2 \mathrm{H})$, $6.92(\mathrm{dd}, J=7.9,1.8,2 \mathrm{H}), 6.91(\mathrm{~d}, J=1.8,2 \mathrm{H}), 6.73(\mathrm{~d}, J=8.5,2 \mathrm{H}), 6.73(\mathrm{~d}, J=7.9$, $2 \mathrm{H}), 6.40(\mathrm{~d}, J=15.7,2 \mathrm{H}), 3.73(\mathrm{~s}, 6 \mathrm{H}), 2.91-2.70(\mathrm{~m}, 4 \mathrm{H}), 2.07-2.02(\mathrm{~m}, 4 \mathrm{H}) ;{ }^{13} \mathrm{C}$ NMR $\left(150 \mathrm{MHz}, \mathrm{DMSO}-d_{6}\right) \delta 165.7,148.7,148.4,148.3,139.1,131.7,127.8,127.0$, $123.8,121.9,119.8,116.2,114.4,113.2,111.6,111.2,111.0,56.0,40.7,25.7 ;{ }^{1} \mathrm{H}$ NMR (400 MHz, $\left.\mathrm{CD}_{3} \mathrm{OD}\right): \delta 7.28(\mathrm{~d}, J=15.7,2 \mathrm{H}), 7.27(\mathrm{~d}, J=8.6,2 \mathrm{H}), 7.00(\mathrm{~d}, J=$ 2.0, 2H), $6.95(\mathrm{~s}, 2 \mathrm{H}), 6.92(\mathrm{dd}, J=8.2,2.0,2 \mathrm{H}), 6.84(\mathrm{~d}, J=8.6,2 \mathrm{H}), 6.72(\mathrm{~d}, J=$ $8.1,2 \mathrm{H}), 6.33(\mathrm{~d}, J=15.7,2 \mathrm{H}), 3.78(\mathrm{~s}, 6 \mathrm{H}), 2.92-2.85(\mathrm{~m}, 2 \mathrm{H}), 2.82-2.74(\mathrm{~m}, 2 \mathrm{H})$, 2.33-2.27 (m, 2H), 2.25-2.18 (m, 2H); ${ }^{13} \mathrm{C}$ NMR (100 MHz, $\left.\mathrm{CD}_{3} \mathrm{OD}\right) \delta=167.5$, $148.3,147.8,147.7,140.2,132.2,127.0,126.9,124.2,121.7,117.6,115.0,112.5$, $112.4,111.3,111.0,110.1,54.9, \quad 40.0,24.9$; HR-ESI-MS $(m / z)$ : calcd. for $\mathrm{C}_{40} \mathrm{H}_{38} \mathrm{~N}_{4} \mathrm{O}_{8} \mathrm{Na}[\mathrm{M}+\mathrm{Na}]^{+}, 725.2582$, found 725.2578 . 
Table S1. Comparison of NMR data (in DMSO- $d_{6}$ ) with synthetic and natural $4,4^{\prime \prime}$-bis( $N$-feruloyl)serotonin $(4)^{[4]}$

\begin{tabular}{|c|c|c|c|c|}
\hline \multirow{2}{*}{ Position } & \multicolumn{2}{|c|}{${ }^{1} \mathrm{H}$, mult., $J(\mathrm{~Hz})$} & \multicolumn{2}{|c|}{${ }^{13} \mathrm{C}$} \\
\hline & Natural & Synthetic & Natural & Synthetic \\
\hline 2 & $6.90, \mathrm{~d}(1.8)$ & $6.90, \mathrm{~d}(1.8)$ & 123.9 & 123.8 \\
\hline 3 & - & - & 113.2 & 113.2 \\
\hline $3 a$ & - & - & 127.9 & 127.8 \\
\hline 4 & - & - & $114.4^{\mathrm{a}}$ & 114.4 \\
\hline 5 & - & - & 148.4 & 148.4 \\
\hline 6 & $6.74, \mathrm{~d}(8.5)$ & $6.73, \mathrm{~d}(8.5)$ & 111.6 & 111.6 \\
\hline 7 & 7.14, d (8.5) & 7.14, d (8.6) & 111.0 & 111.0 \\
\hline $7 \mathrm{a}$ & - & - & 131.7 & 131.7 \\
\hline$\beta-\mathrm{CH}_{2}$ & $2.07, \mathrm{~m}$ & $2.07-2.03, \mathrm{~m}$ & 25.7 & 25.7 \\
\hline$\alpha-\mathrm{CH}_{2}$ & $2.80, \mathrm{~m}$ & 2.91-2.70, m & 40.7 & 40.7 \\
\hline $1^{\prime}$ & - & - & $127.1^{\mathrm{b}}$ & 127.0 \\
\hline $2^{\prime}$ & 7.02, d (1.8) & $7.02, \mathrm{~d}(1.8)$ & 111.3 & 111.2 \\
\hline $3^{\prime}$ & - & - & 148.4 & 148.3 \\
\hline $4^{\prime}$ & - & - & 148.8 & 148.7 \\
\hline $5^{\prime}$ & $6.74, \mathrm{~d}(7.9)$ & $6.73, \mathrm{~d}(7.9)$ & 116.2 & 116.2 \\
\hline $6^{\prime}$ & $6.90, \mathrm{dd}(7.9,1.8)$ & $6.90, \mathrm{dd}(7.9,1.8)$ & 122.0 & 122.0 \\
\hline $7^{\prime}$ & $7.21, \mathrm{~d}(15.8)$ & 7.21, d (15.6) & 139.2 & 139.1 \\
\hline $8^{\prime}$ & $6.40, \mathrm{~d}(15.8)$ & $6.40, \mathrm{~d}(15.7)$ & 119.8 & 119.8 \\
\hline $9^{\prime}$ & - & - & 165.8 & 165.7 \\
\hline $\mathrm{OMe}$ & $3.72, \mathrm{~s}$ & $3.73, \mathrm{~s}$ & 56.1 & 56.0 \\
\hline NH & $10.4, \mathrm{~s}$ & $10.42, \mathrm{~s}$ & & \\
\hline $\mathrm{CONH}$ & $7.38, \mathrm{t}$ & $7.37, \mathrm{t}(4.9)$ & & \\
\hline $5-\mathrm{OH}$ & $7.75, \mathrm{~s}$ & $7.75, \mathrm{~s}$ & & \\
\hline $4^{\prime}-\mathrm{OH}$ & $9.4, \mathrm{br}$ & $9.38, \mathrm{br}$ & & \\
\hline
\end{tabular}

${ }^{\mathrm{a}}$ In the original reports ${ }^{[4]}$, the ${ }^{13} \mathrm{C}$ NMR of C-4 was mistyped as 114.0 instead of 114.4.

${ }^{\mathrm{b}} \mathrm{The}{ }^{13} \mathrm{C}$ NMR of $\mathrm{C}-1^{\prime}$ was originally assigned as $125.0 \mathrm{ppm}^{[4]}$. However, this signal was found from some impurities and the peak at $127.1 \mathrm{ppm}$ was the genuine signal of $\mathrm{C}-1^{\prime}$. 
Table S2. Comparison of NMR data (in $\mathrm{CD}_{3} \mathrm{OD}$ ) with synthetic $4,4^{\prime \prime}$-bis( $N$-feruloyl)serotonin (4) and natural montamine $(5)^{[5]}$

\begin{tabular}{|c|c|c|c|c|}
\hline \multirow{3}{*}{ Position } & \multicolumn{2}{|c|}{${ }^{1} \mathrm{H}$, mult., $J(\mathrm{~Hz})$} & \multicolumn{2}{|r|}{${ }^{13} \mathrm{C}$} \\
\hline & \multicolumn{3}{|c|}{ Synthetic } & \multirow{2}{*}{$\begin{array}{c}\text { Synthetic } \\
4,4^{\prime \prime} \text {-bis }(N \text { - feruloyl }) \\
\text { serotonin }(\mathbf{4})\end{array}$} \\
\hline & Montamine (5) & $\begin{array}{c}4,4^{\prime \prime}-\text { bis }(N \text { - feruloyl }) \\
\text { serotonin }(\mathbf{4})\end{array}$ & Montamine (5) & \\
\hline 2 & $6.93, \mathrm{~s}$ & $6.95, \mathrm{~s}$ & 126.0 & 124.2 \\
\hline 3 & - & - & 111.0 & 111.0 \\
\hline $3 a$ & - & - & 129.5 & 127.0 \\
\hline 4 & $6.83, \mathrm{~d}(2.0)$ & - & 111.2 & 112.4 \\
\hline 5 & - & - & 148.0 & 147.8 \\
\hline 6 & $6.82, \mathrm{dd}(8.0,2.0)$ & $6.84, \mathrm{~d}(8.0)$ & 111.1 & 111.3 \\
\hline 7 & $7.26, \mathrm{~d}(8.0)$ & $7.27, \mathrm{~d}(8.0)$ & 111.5 & 112.5 \\
\hline $7 \mathrm{a}$ & - & - & 133.0 & 132.1 \\
\hline \multirow[t]{2}{*}{$\beta-\mathrm{CH}_{2}$} & $2.27, \mathrm{~m}$ & $2.33-2.27, \mathrm{~m}$ & 25.0 & 24.9 \\
\hline & $2.19, \mathrm{~m}$ & $2.25-2.18, \mathrm{~m}$ & & \\
\hline \multirow[t]{2}{*}{$\alpha-\mathrm{CH}_{2}$} & $2.86, \mathrm{~m}$ & $2.92-2.85, \mathrm{~m}$ & 41.0 & 40.0 \\
\hline & $2.77, \mathrm{~m}$ & $2.82-2.74, \mathrm{~m}$ & & \\
\hline $1^{\prime}$ & - & - & 129.0 & 126.9 \\
\hline $2^{\prime}$ & $6.99, \mathrm{~d}(2.0)$ & $7.00, \mathrm{~d}(2.0)$ & 110.0 & 110.0 \\
\hline $3^{\prime}$ & - & - & 146.0 & 147.7 \\
\hline $4^{\prime}$ & - & - & 149.0 & 148.3 \\
\hline $5^{\prime}$ & $6.70, \mathrm{~d}(8.4)$ & $6.72, \mathrm{~d}(8.2)$ & 115.0 & 115.0 \\
\hline $6^{\prime}$ & $6.91, \mathrm{dd}(8.4,2.0)$ & $6.92, \mathrm{dd}(8.2,2.0)$ & 121.0 & 121.7 \\
\hline $7^{\prime}$ & 7.27, d (15.6) & $7.28, \mathrm{~d}(15.7)$ & 141.0 & 140.1 \\
\hline $8^{\prime}$ & 6.31, d (15.6) & $6.33, d(15.7)$ & 117.5 & 117.6 \\
\hline $9^{\prime}$ & - & - & 172.0 & 167.5 \\
\hline $\mathrm{OMe}$ & $3.78, \mathrm{~s}$ & $3.78, \mathrm{~s}$ & 55.5 & 54.9 \\
\hline
\end{tabular}

\section{SupplementaryReferences:}

[1] Silva-Lopez, E. I.; Barden, A. O.; Brozik, J. A. Bioorg. Med. Chem. Lett. 2013, $23,773$.

[2] Koizumi, Y.; Kobayashi, H.; Wakimoto, T.; Furuta, T.; Fukuyama, T.; Kan, T. J. Am. Chem. Soc. 2008, 130, 16854.

[3] Sun, D.; Zhao, Q.; Li, C. Org. Lett. 2011, 13, 5302.

[4] (a) Zhang, H. L.; Nagatsu, A.; Sakakibara, J. Chem. Pharm. Bull. 1996, 44, 874. (b) Zhang, H. L.; Nagatsu, A.; Watanabe, T.; Sakakibara, J.; Okuyama, H. Chem. Pharm. Bull. 1997, 45, 1910.

[5] Shoeb, M.; MacManus, S. M.; Jaspars, M.; Trevidu, J.; Nahar, L.; Kong-Thoo-Lin, P.; Sarker, S. D. Tetrahedron 2006, 62, 11172. 


\section{Supplementary Figures}
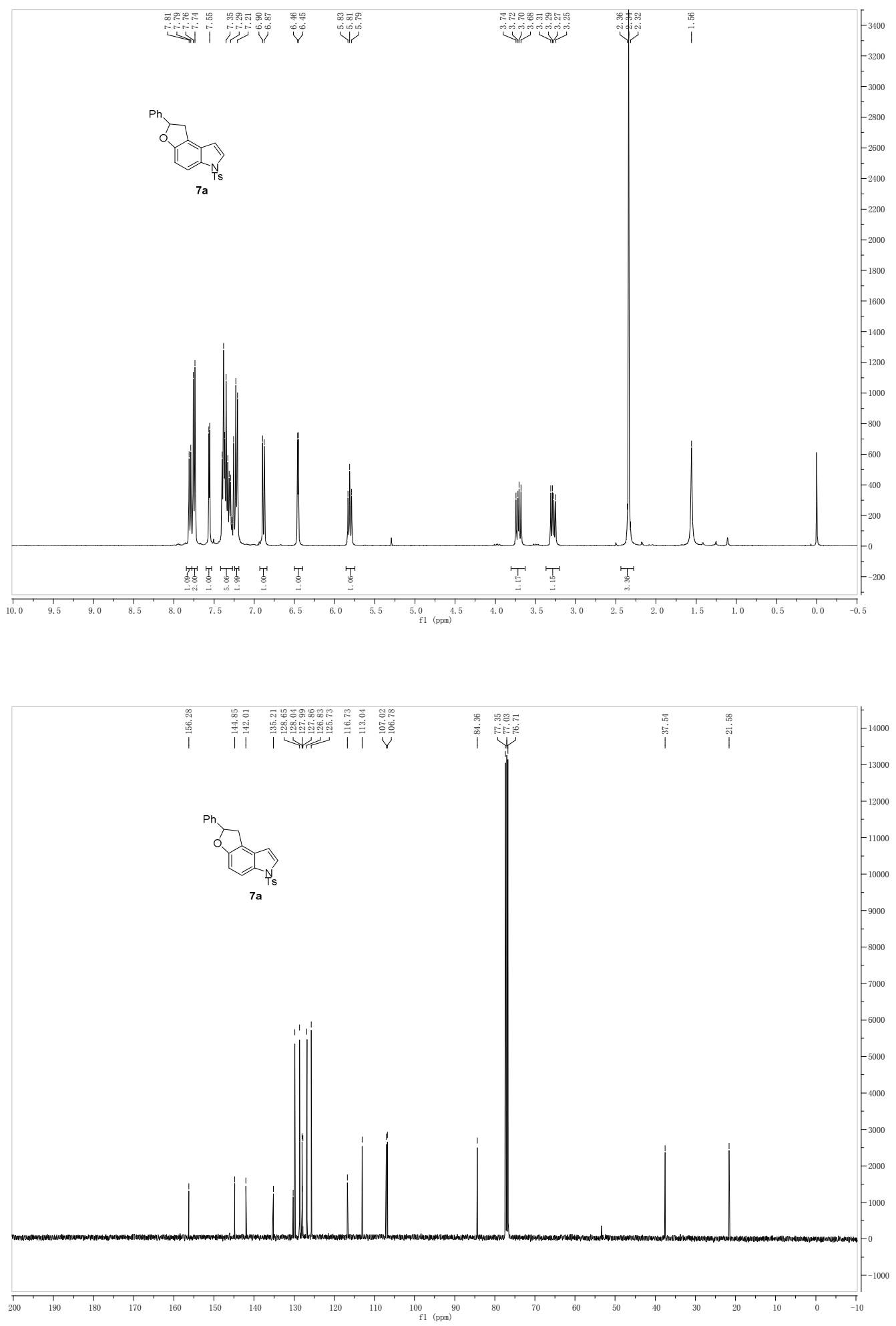

Supplementary Figure 1. ${ }^{1} \mathrm{H}$ and ${ }^{13} \mathrm{C}$ NMR spectra for $7 \mathrm{a}$. 

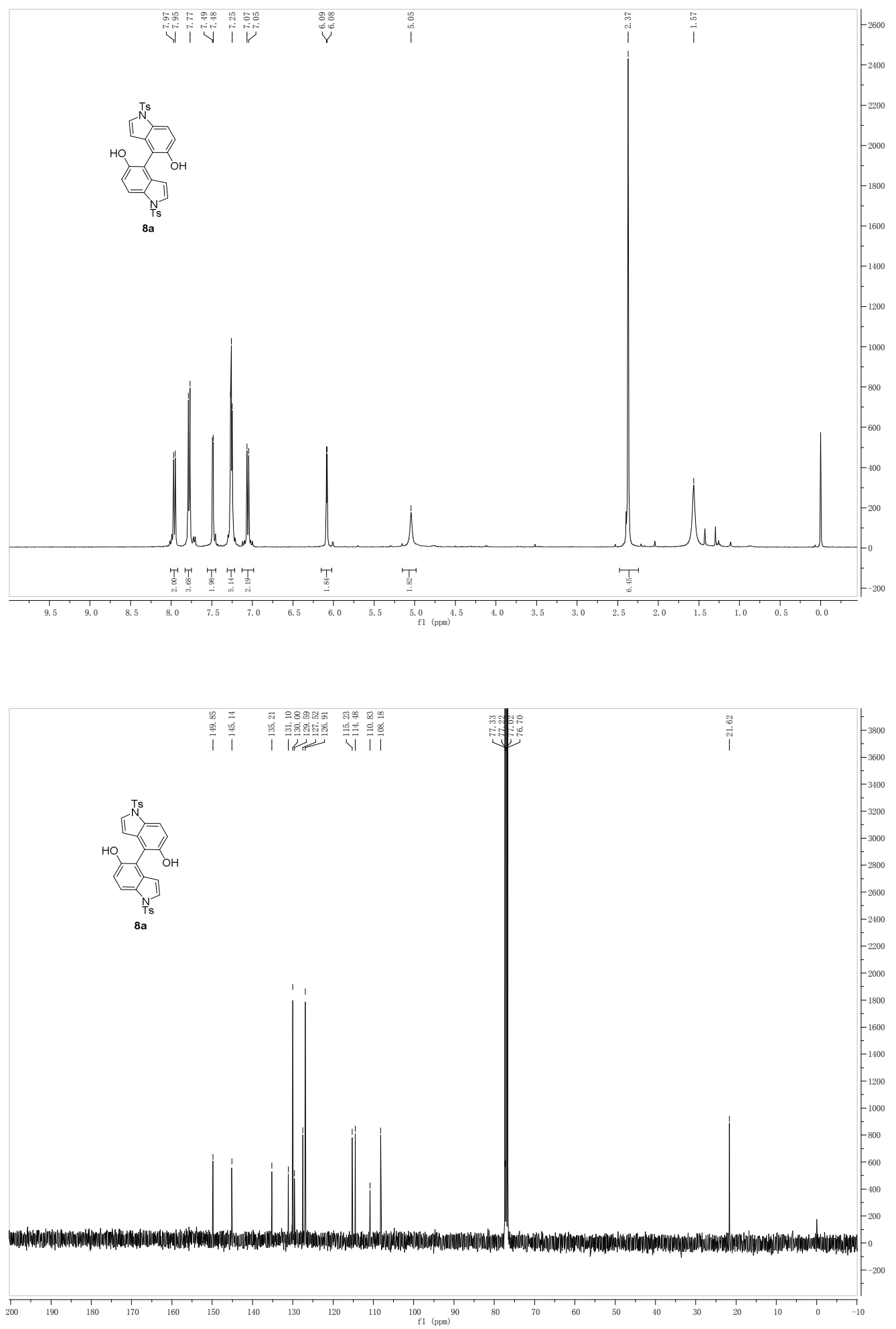

Supplementary Figure 2. ${ }^{1} \mathrm{H}$ and ${ }^{13} \mathrm{C}$ NMR spectra for 8 . 

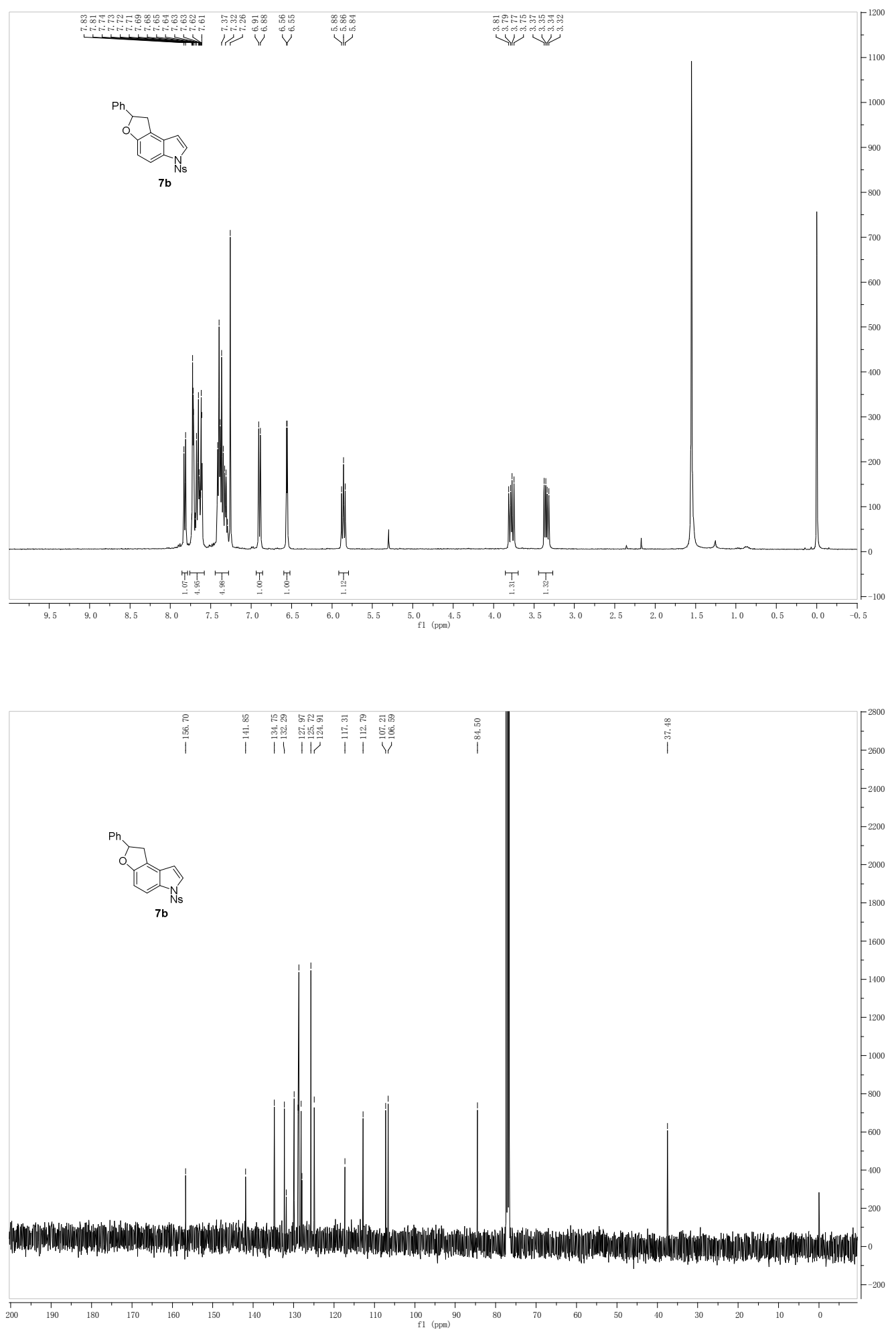

Supplementary Figure 3. ${ }^{1} \mathrm{H}$ and ${ }^{13} \mathrm{C}$ NMR spectra for $7 \mathrm{~b}$. 

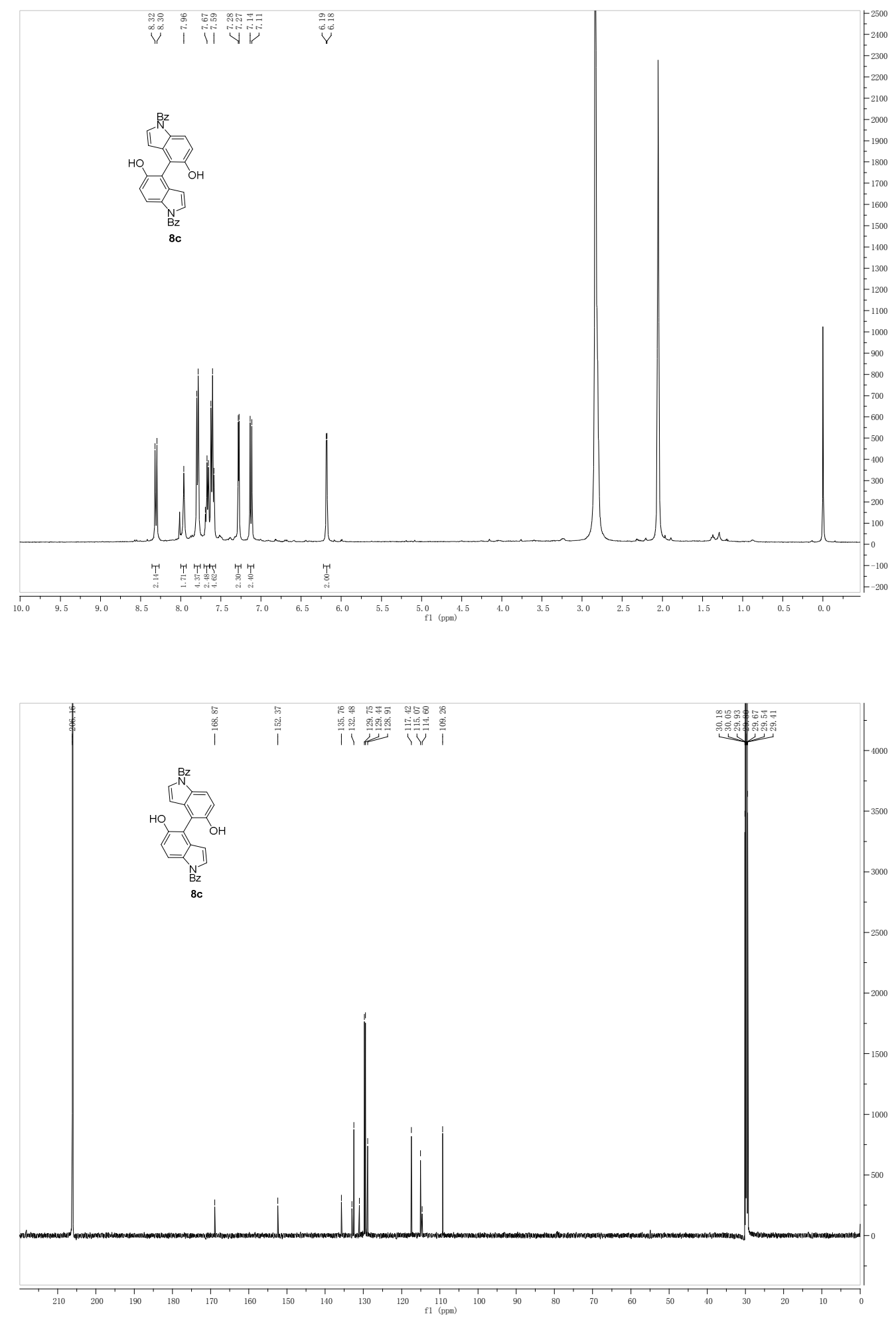

Supplementary Figure 4. ${ }^{1} \mathrm{H}$ and ${ }^{13} \mathrm{C}$ NMR spectra for $8 \mathrm{c}$. 

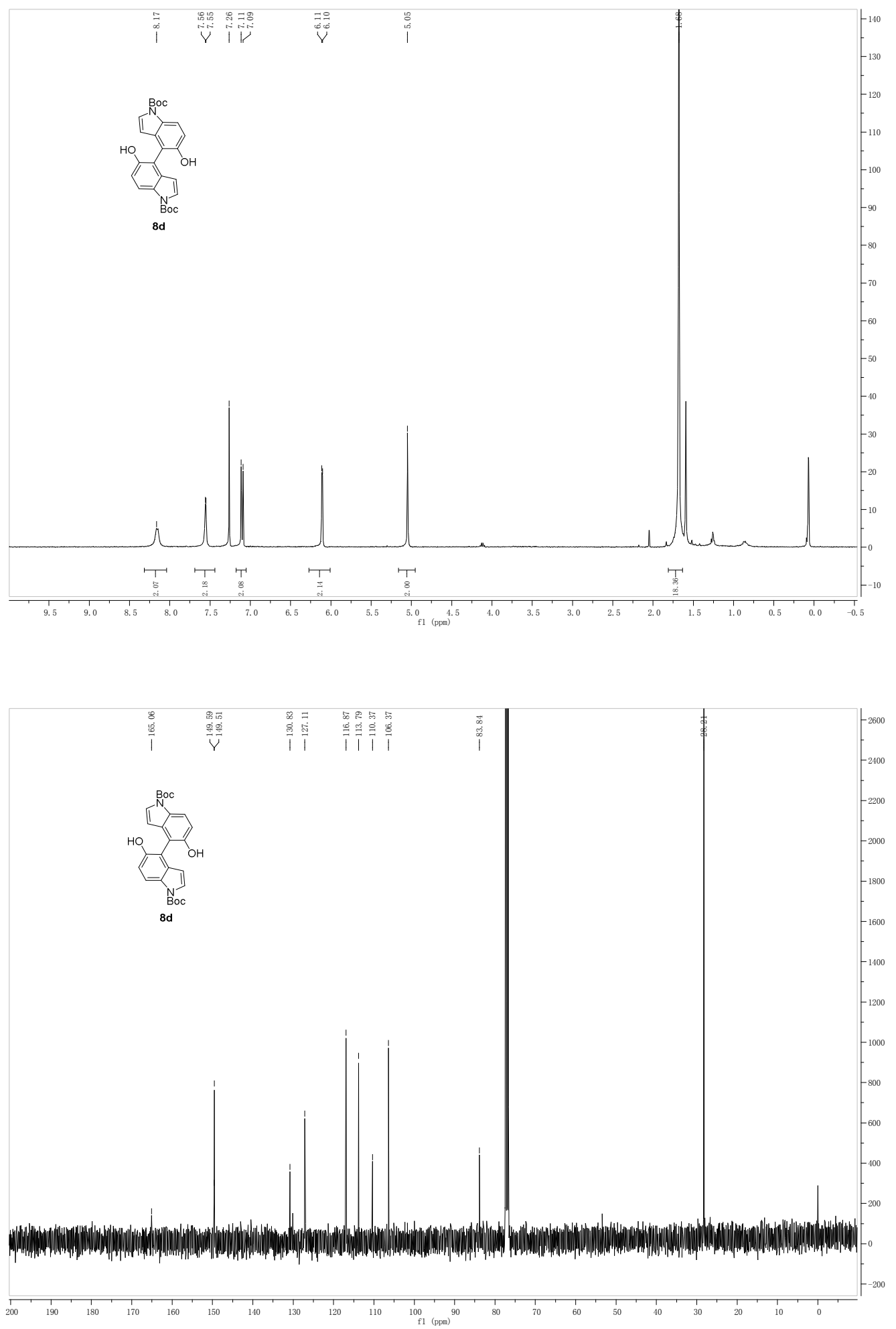

Supplementary Figure 5. ${ }^{1} \mathrm{H}$ and ${ }^{13} \mathrm{C}$ NMR spectra for $8 \mathrm{~d}$. 

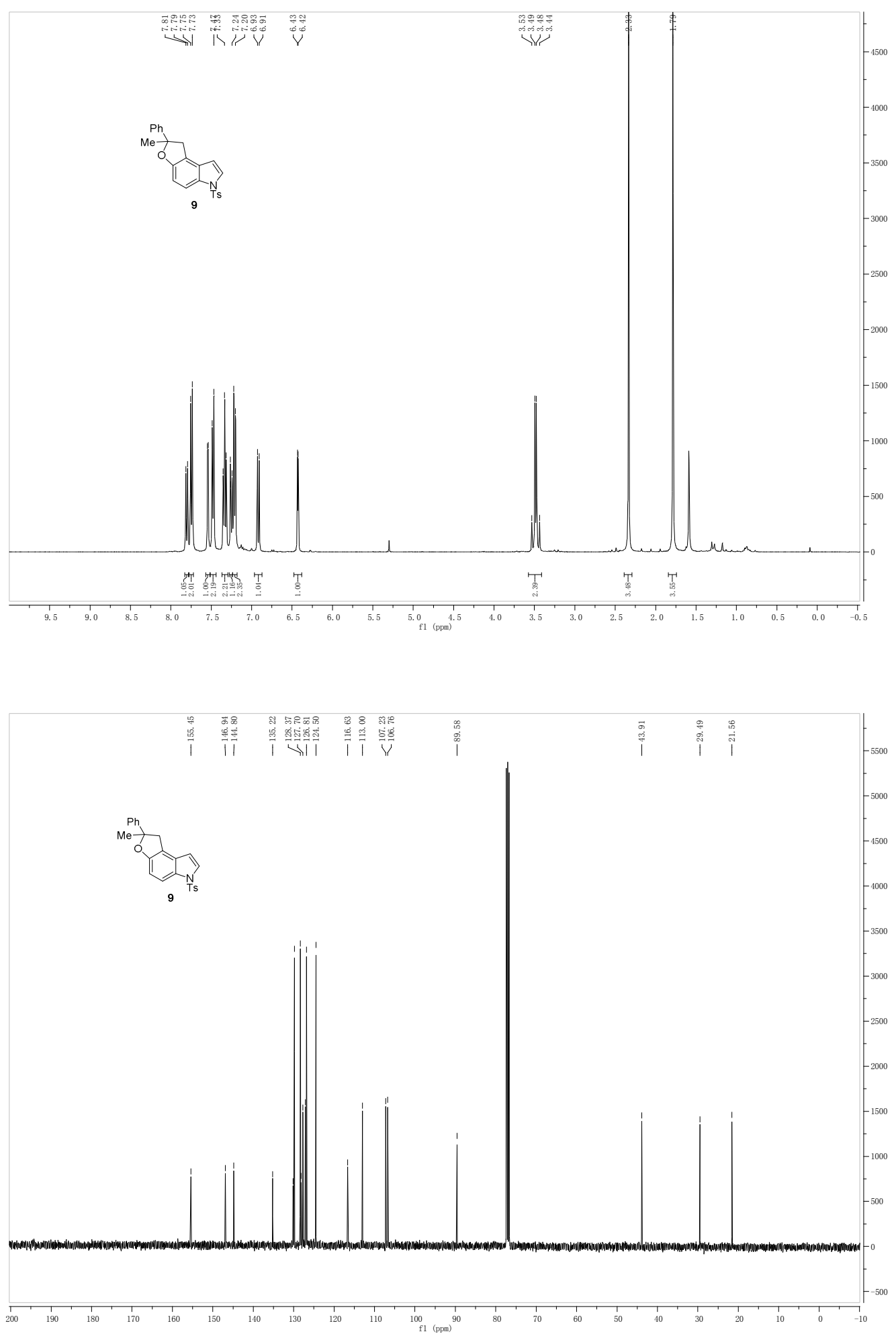

Supplementary Figure 6. ${ }^{1} \mathrm{H}$ and ${ }^{13} \mathrm{C}$ NMR spectra for 9. 

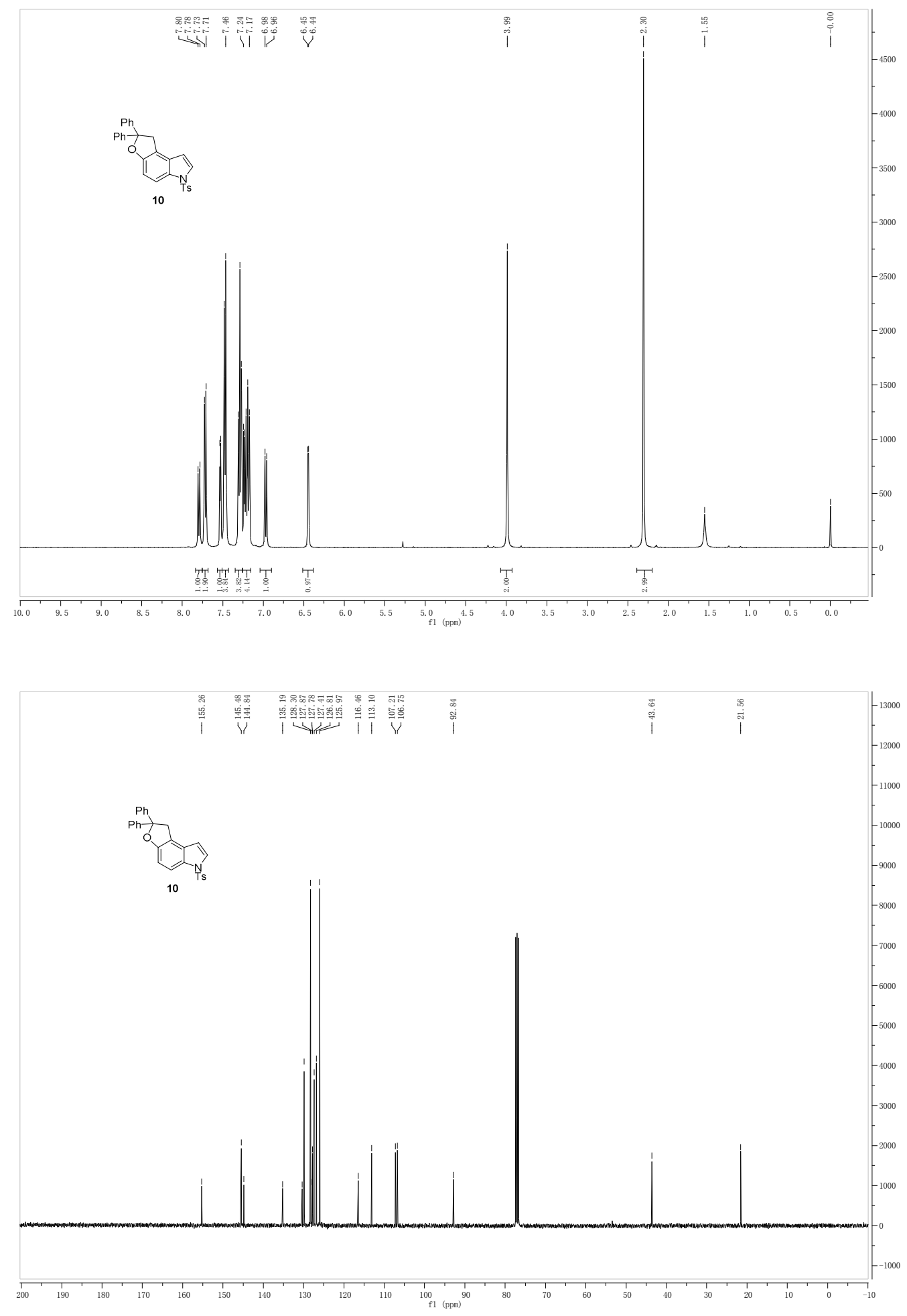

Supplementary Figure 7. ${ }^{1} \mathrm{H}$ and ${ }^{13} \mathrm{C}$ NMR spectra for 10. 

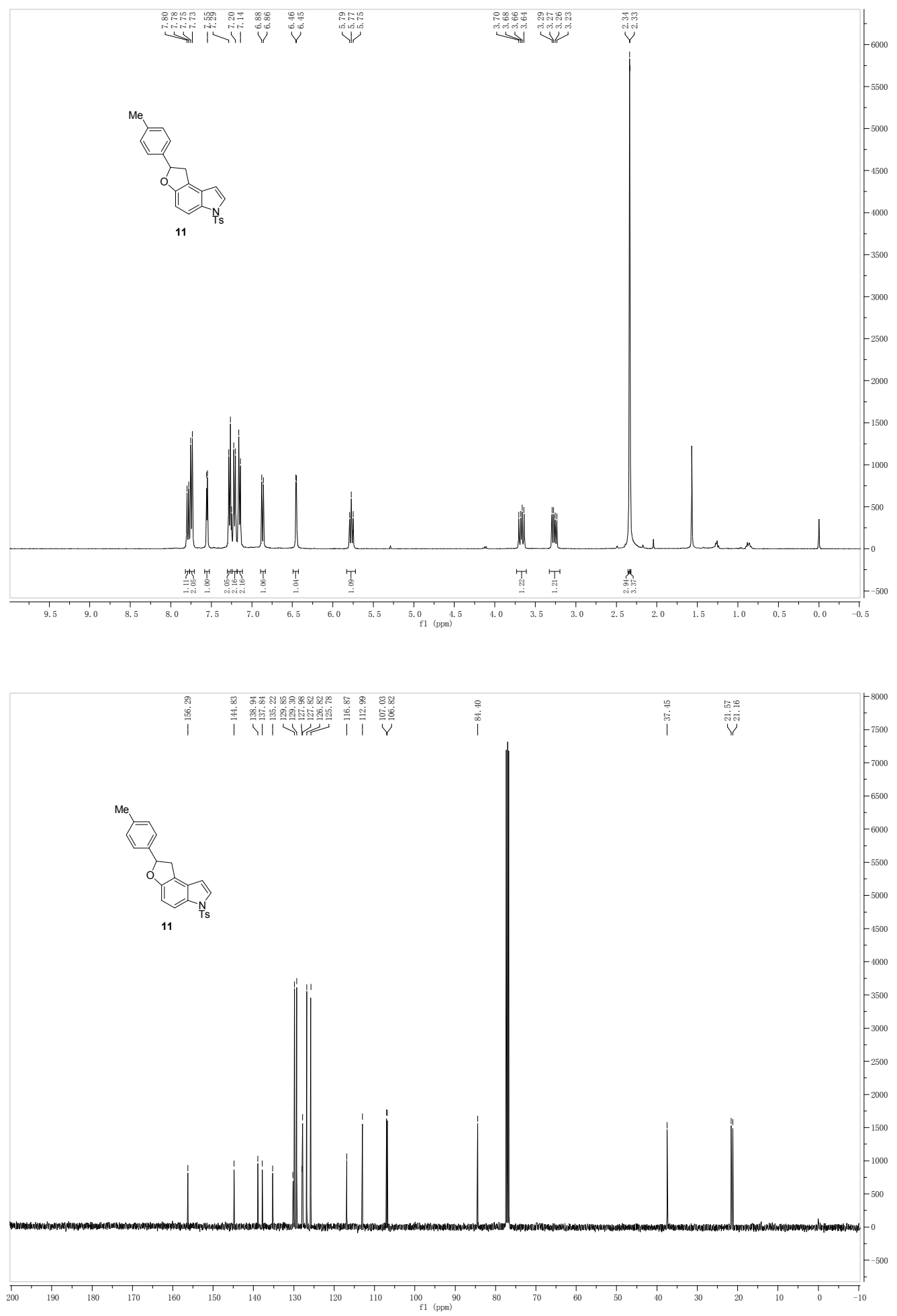

Supplementary Figure 8. ${ }^{1} \mathrm{H}$ and ${ }^{13} \mathrm{C}$ NMR spectra for 11. 

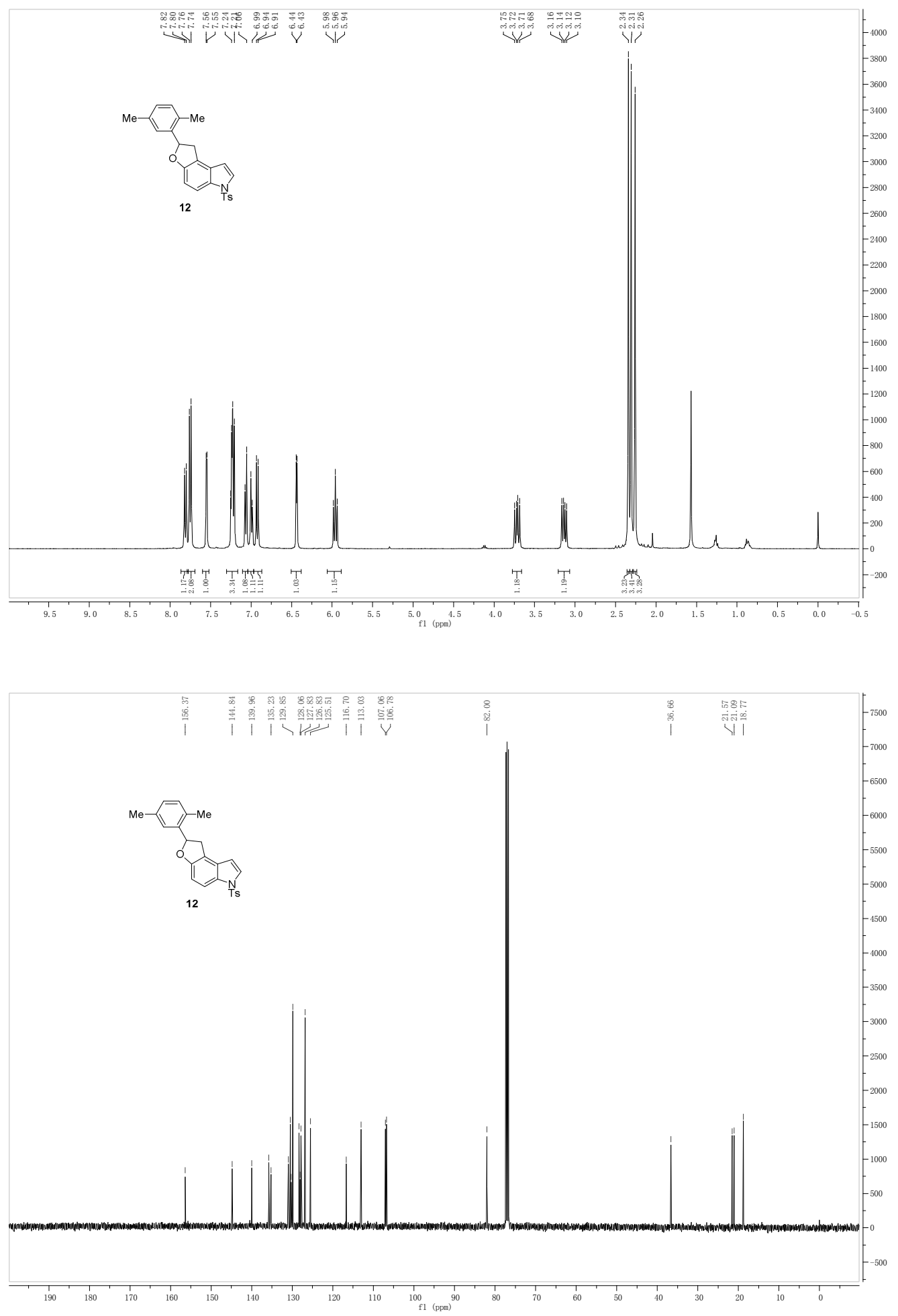

Supplementary Figure 9. ${ }^{1} \mathrm{H}$ and ${ }^{13} \mathrm{C}$ NMR spectra for 12. 

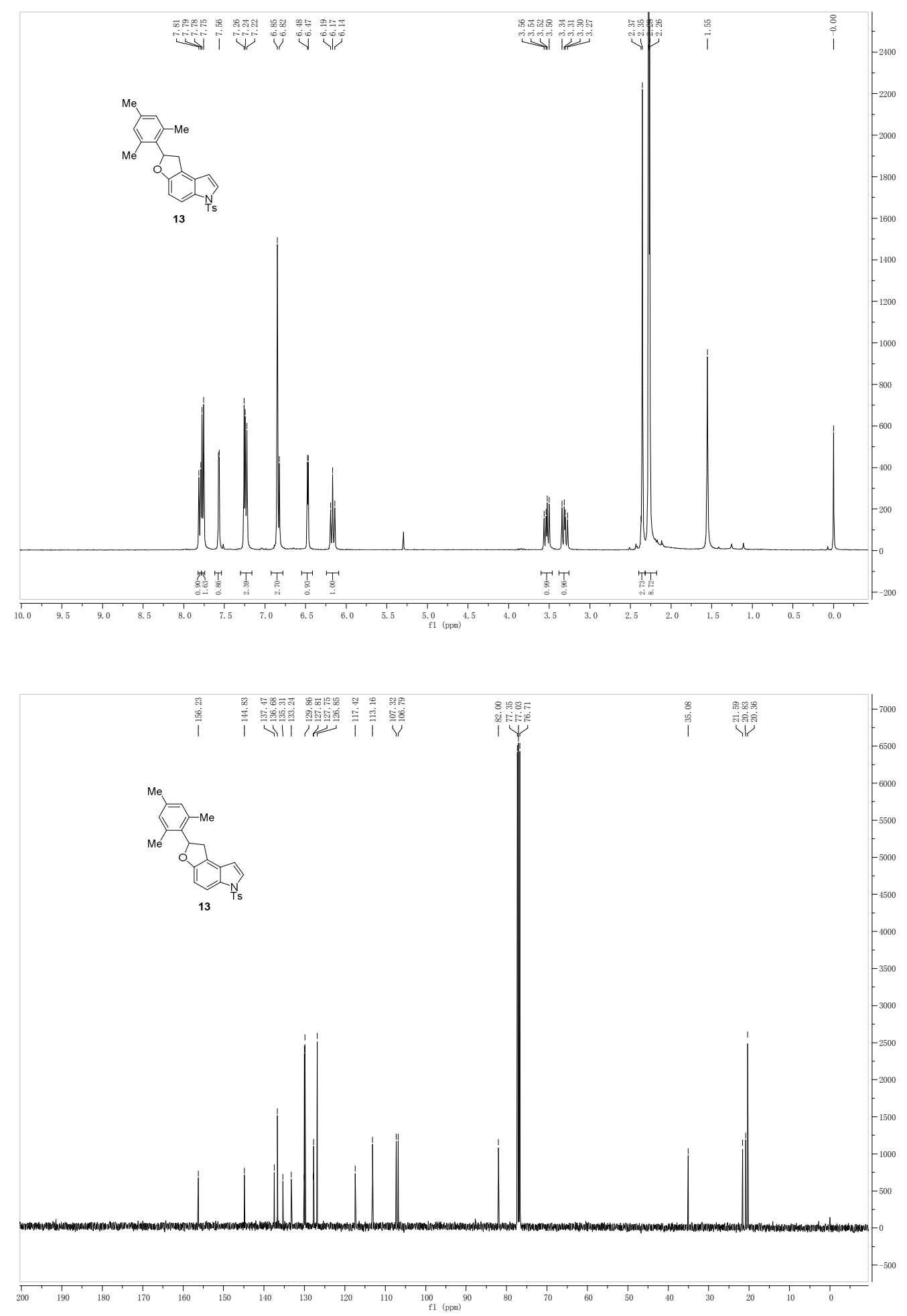

Supplementary Figure 10. ${ }^{1} \mathrm{H}$ and ${ }^{13} \mathrm{C}$ NMR spectra for 13. 

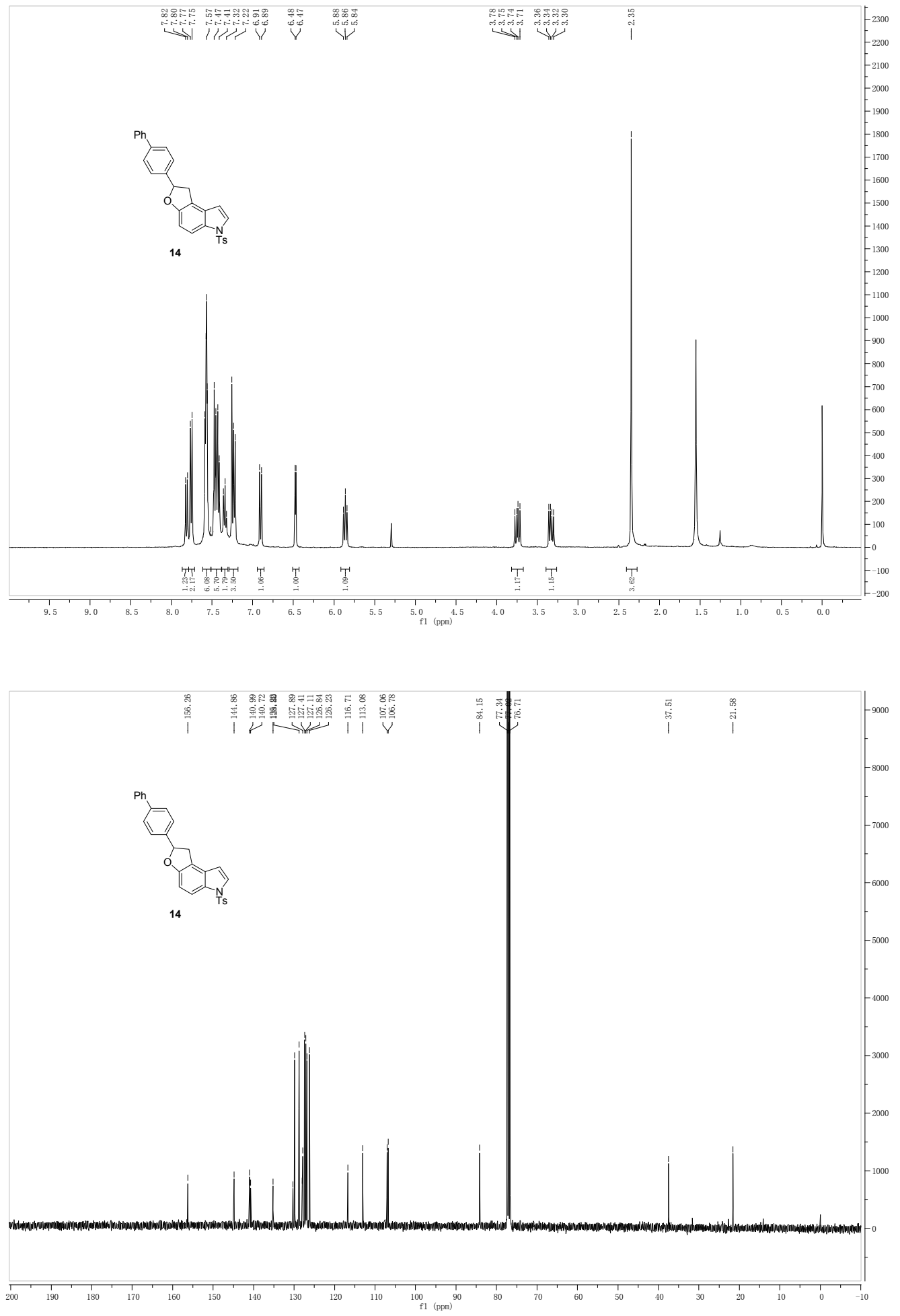

Supplementary Figure 11. ${ }^{1} \mathrm{H}$ and ${ }^{13} \mathrm{C}$ NMR spectra for 14. 

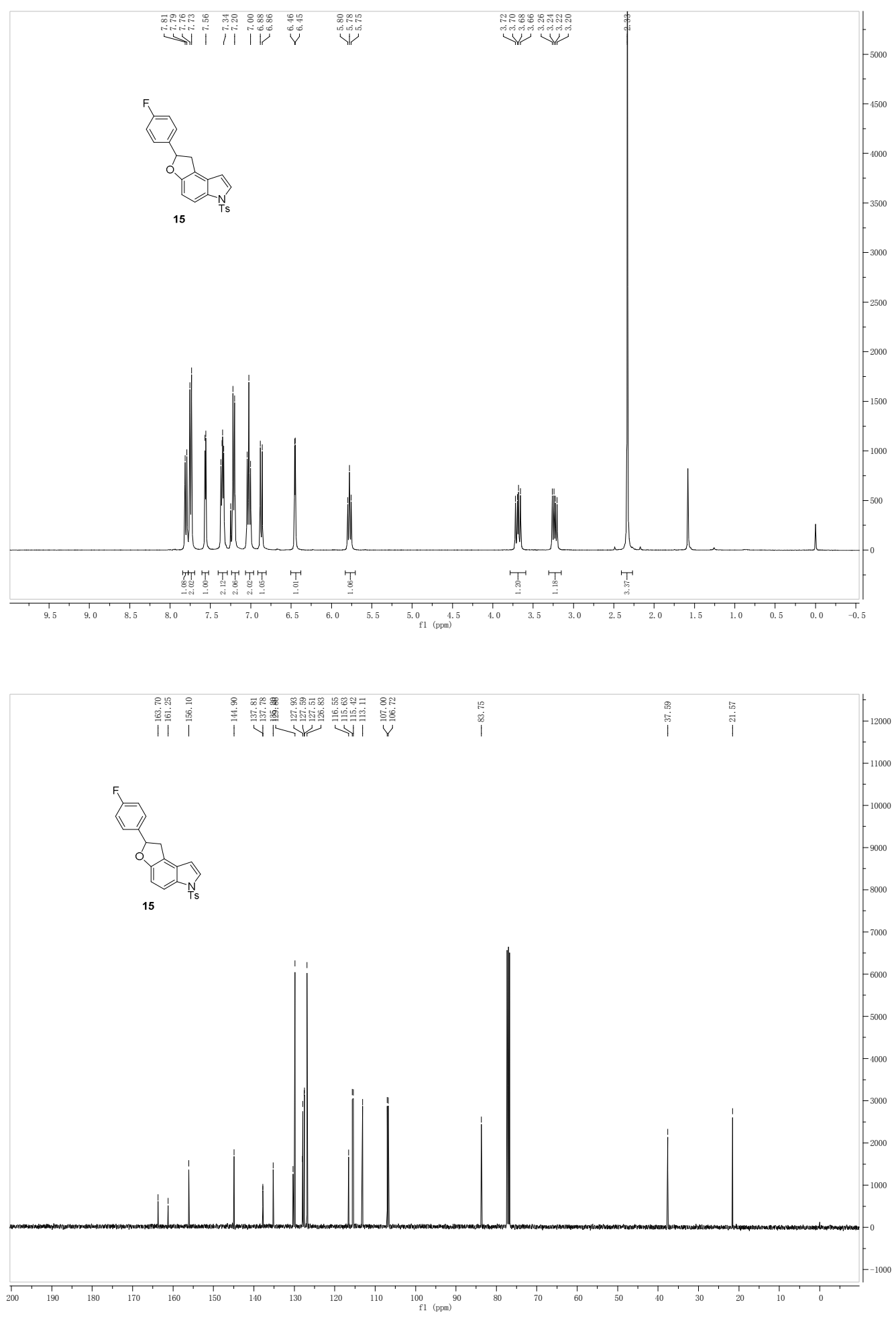

Supplementary Figure 12. ${ }^{1} \mathrm{H}$ and ${ }^{13} \mathrm{C}$ NMR spectra for 15. 

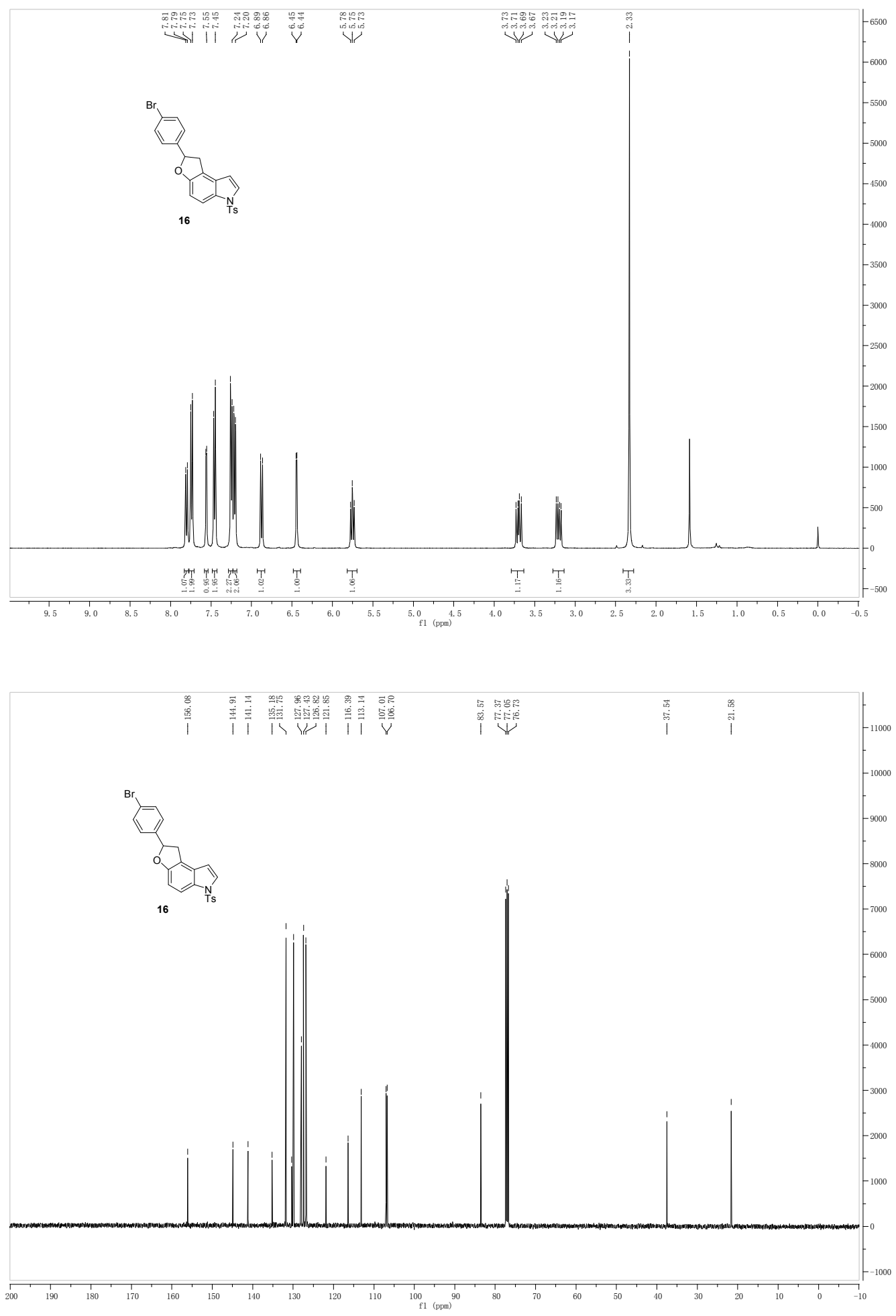

Supplementary Figure 13. ${ }^{1} \mathrm{H}$ and ${ }^{13} \mathrm{C}$ NMR spectra for 16. 

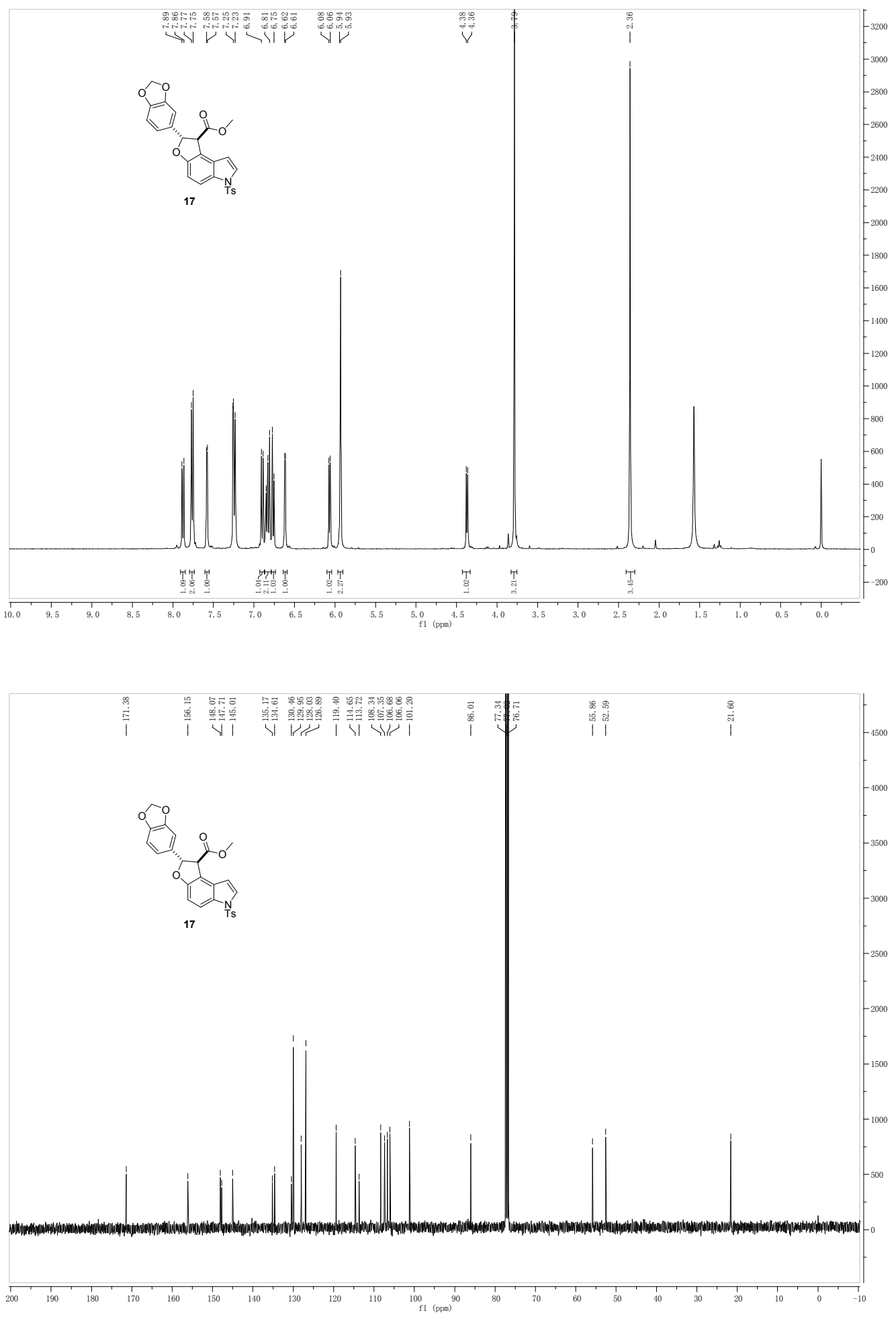

Supplementary Figure 14. ${ }^{1} \mathrm{H}$ and ${ }^{13} \mathrm{C}$ NMR spectra for 17. 

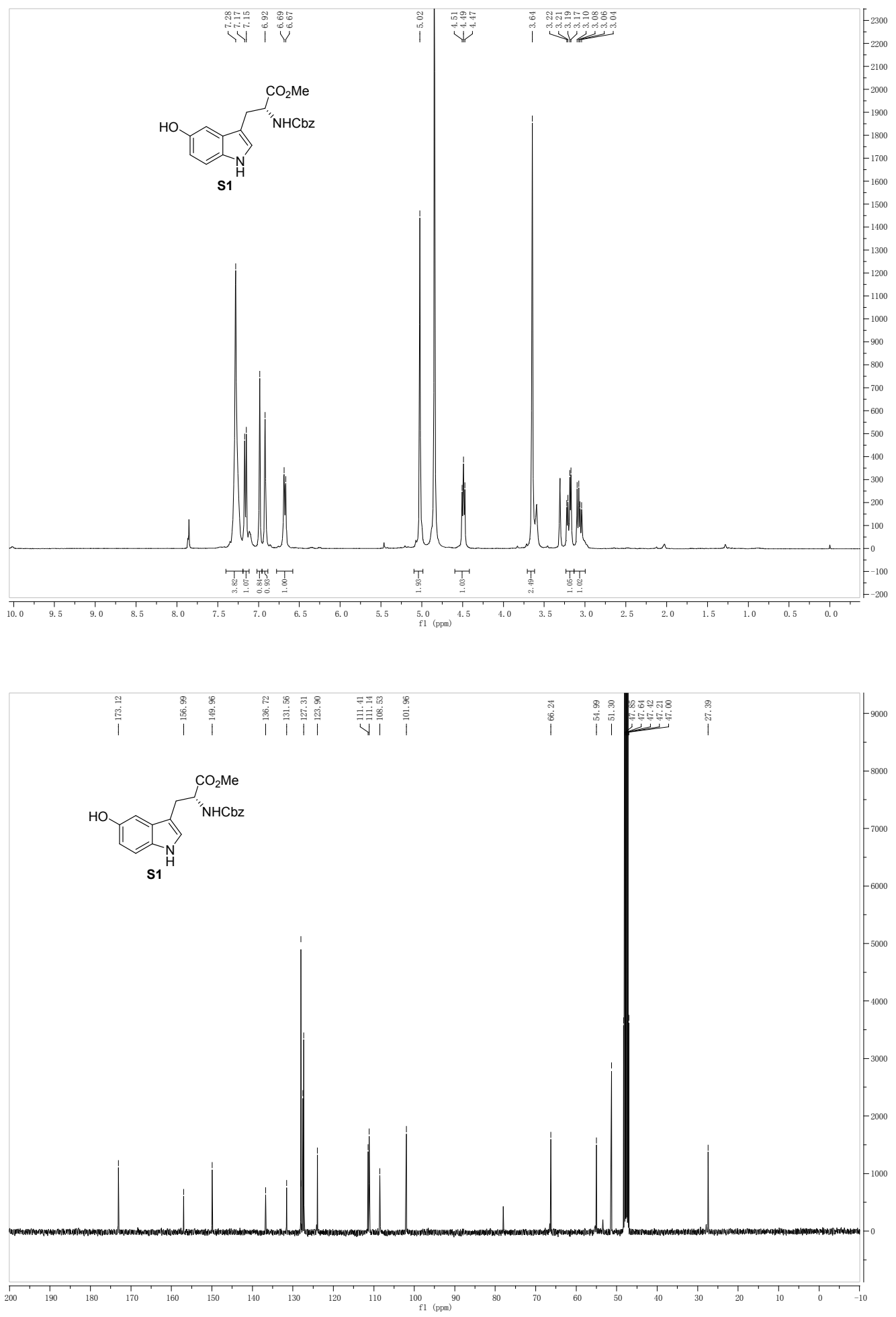

Supplementary Figure 15. ${ }^{1} \mathrm{H}$ and ${ }^{13} \mathrm{C}$ NMR spectra for S1. 

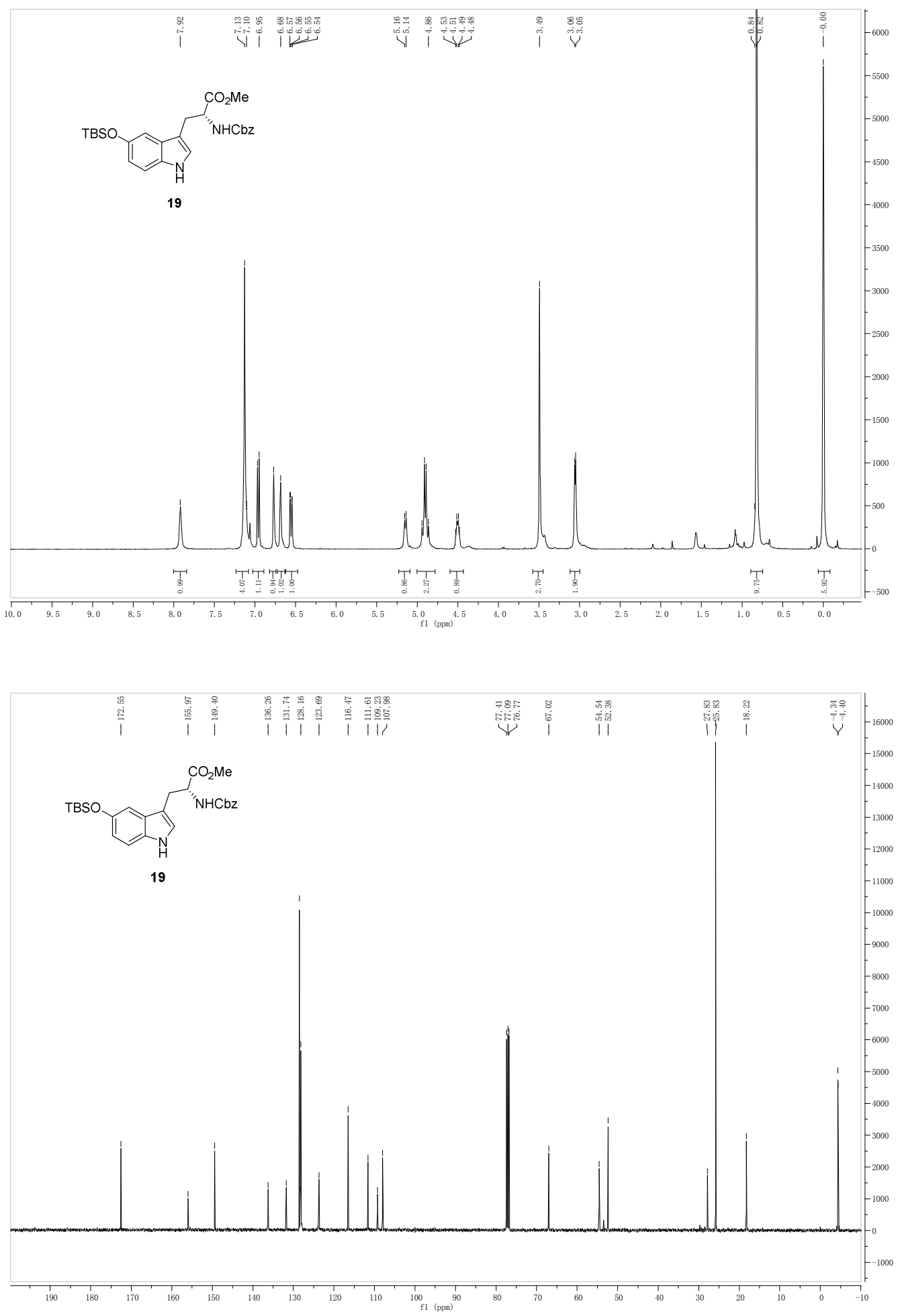

Supplementary Figure 16. ${ }^{1} \mathrm{H}$ and ${ }^{13} \mathrm{C}$ NMR spectra for 19. 

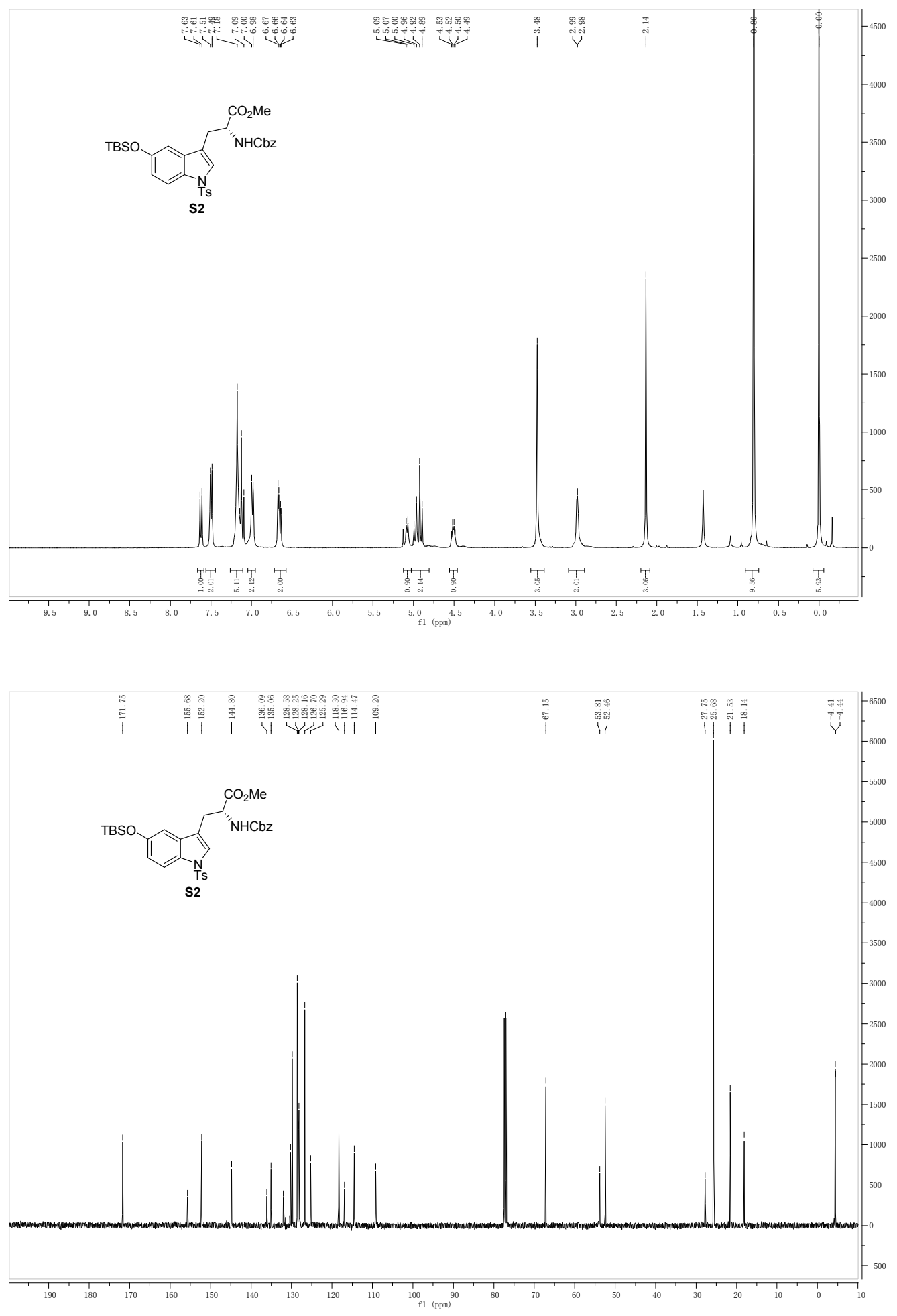

Supplementary Figure 17. ${ }^{1} \mathrm{H}$ and ${ }^{13} \mathrm{C}$ NMR spectra for S2. 

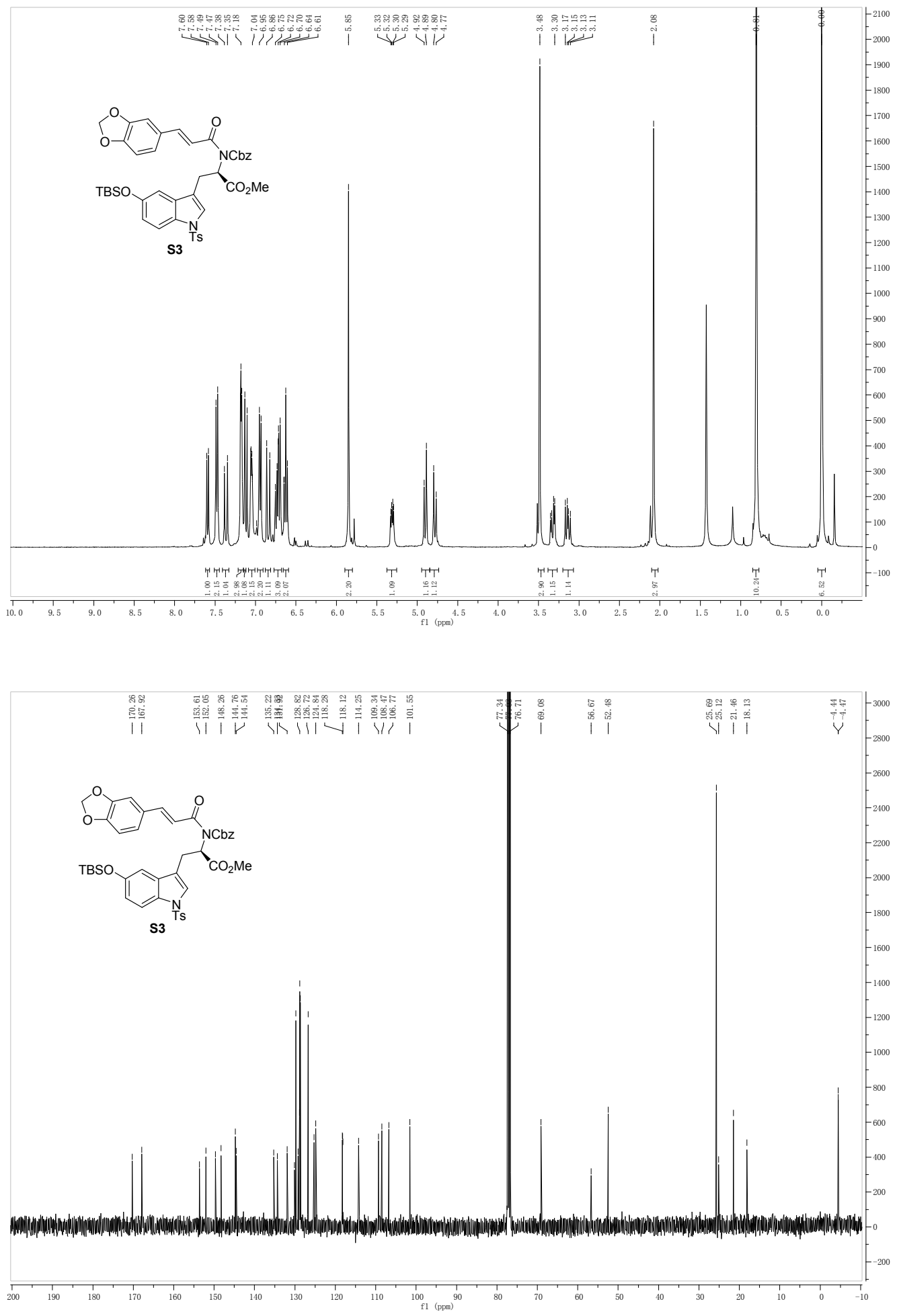

Supplementary Figure 18. ${ }^{1} \mathrm{H}$ and ${ }^{13} \mathrm{C}$ NMR spectra for S3. 

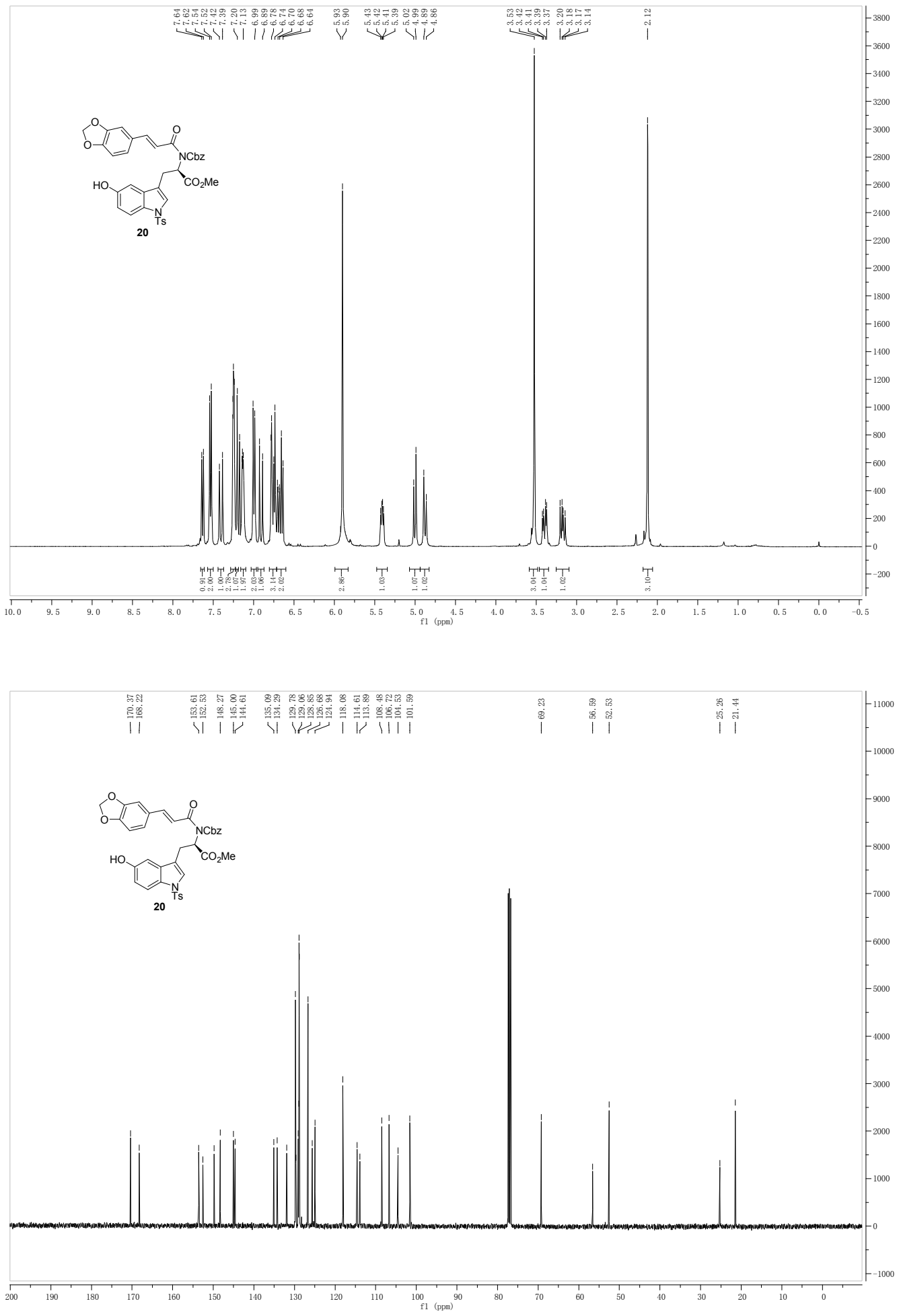

Supplementary Figure 19. ${ }^{1} \mathrm{H}$ and ${ }^{13} \mathrm{C}$ NMR spectra for 20. 

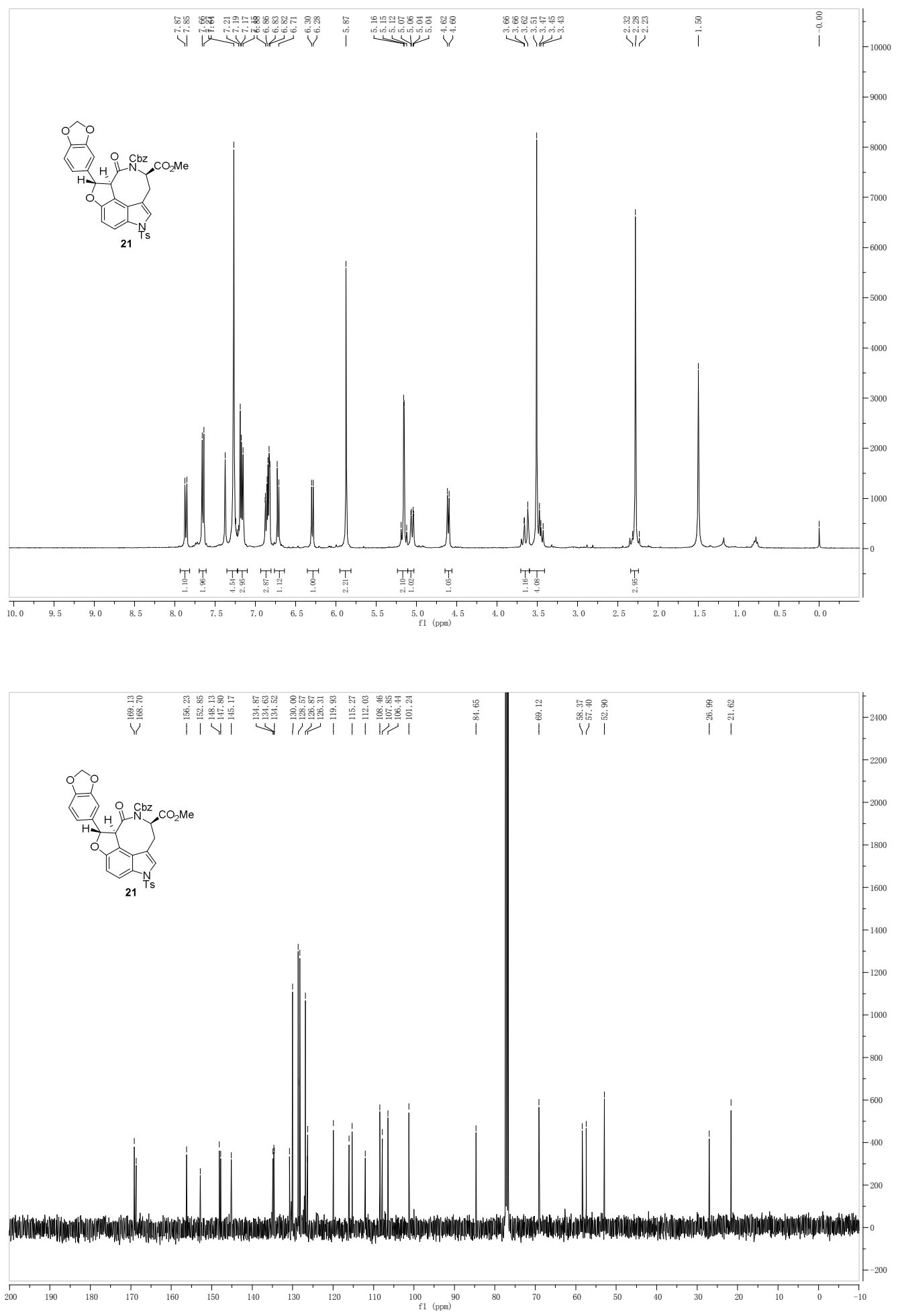

Supplementary Figure 20. ${ }^{1} \mathrm{H}$ and ${ }^{13} \mathrm{C}$ NMR spectra for 21. 

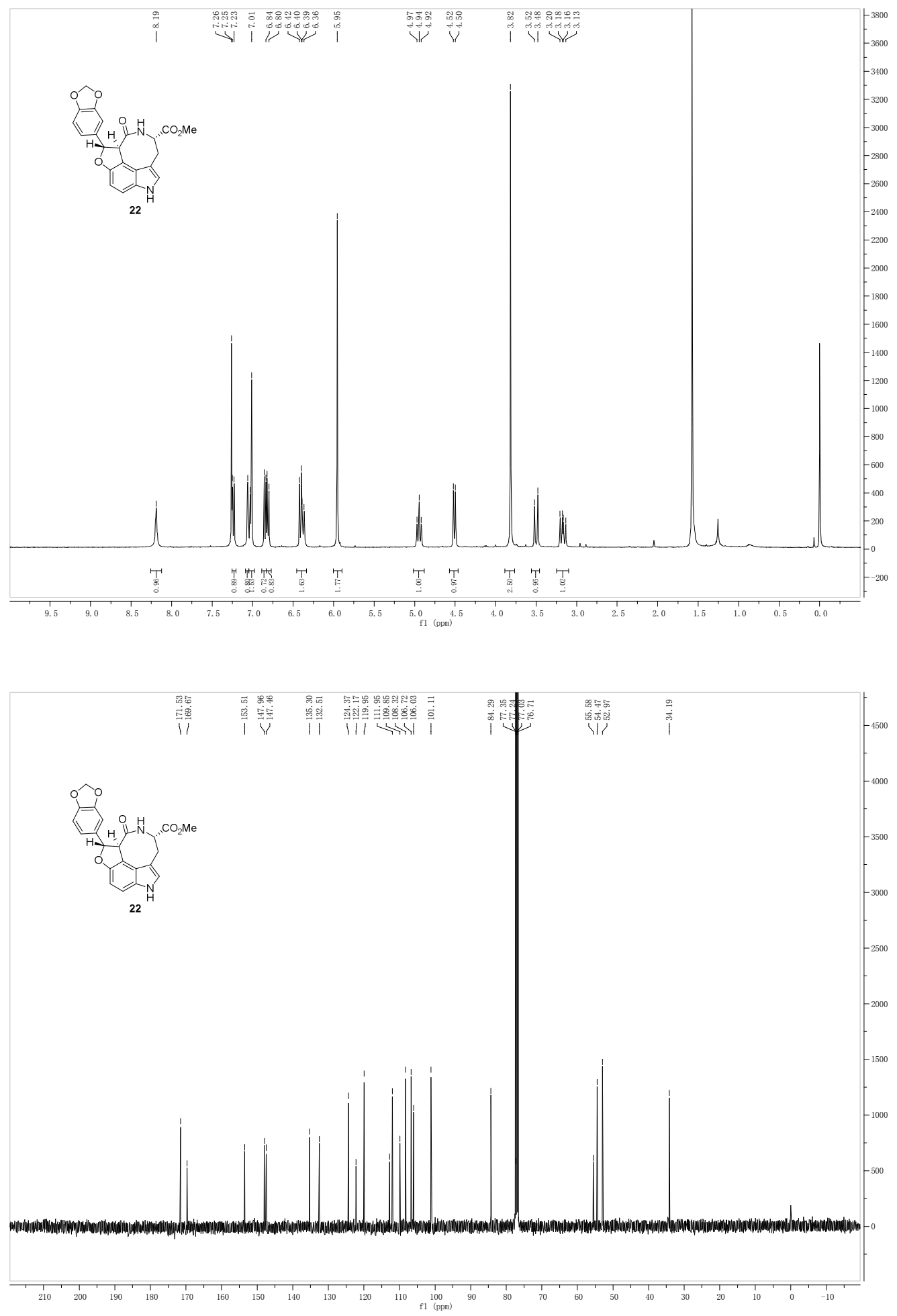

Supplementary Figure 21. ${ }^{1} \mathrm{H}$ and ${ }^{13} \mathrm{C}$ NMR spectra for 22. 

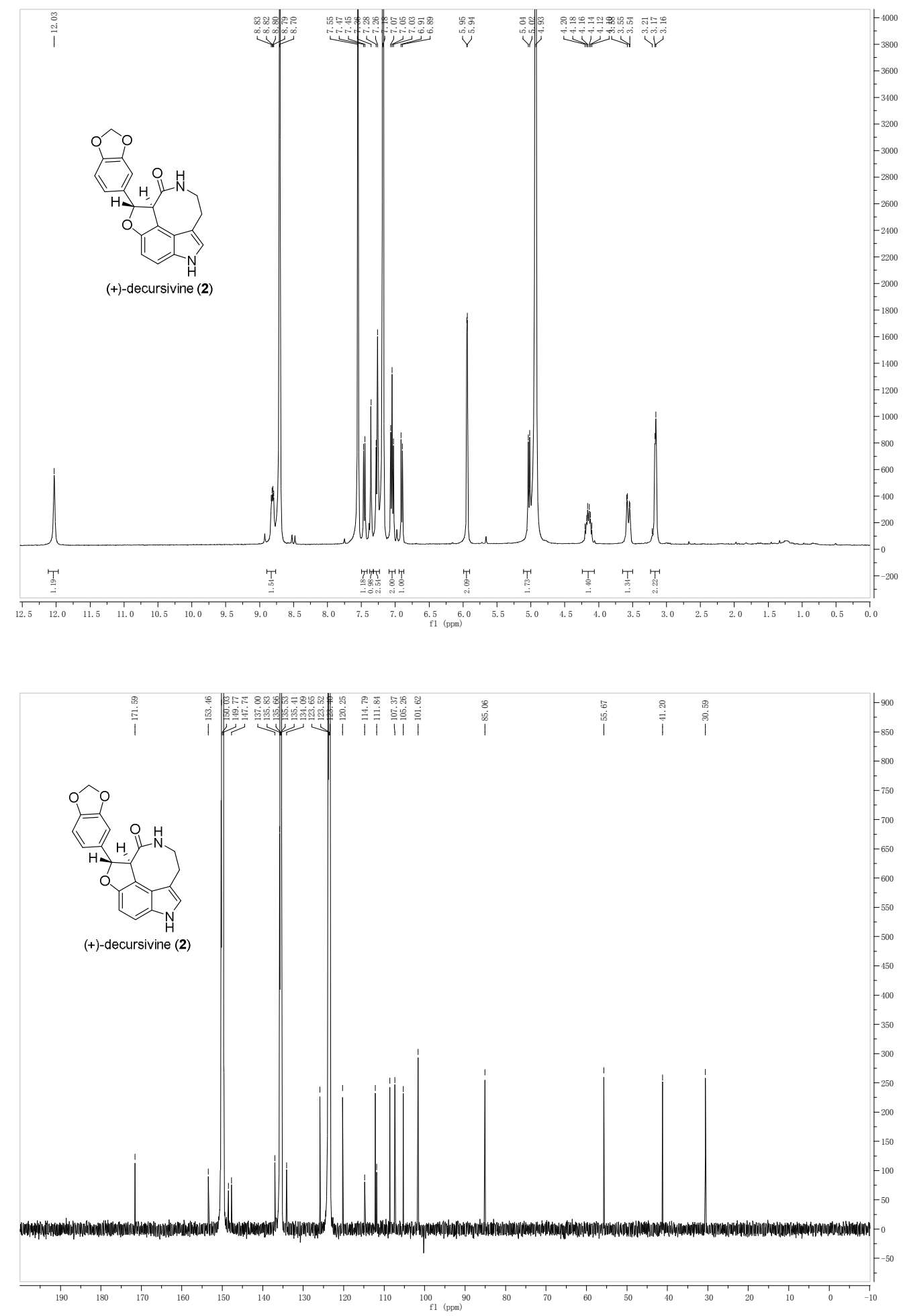

Supplementary Figure 22. ${ }^{1} \mathrm{H}$ and ${ }^{13} \mathrm{C}$ NMR spectra for (+)-decursivine (2). 

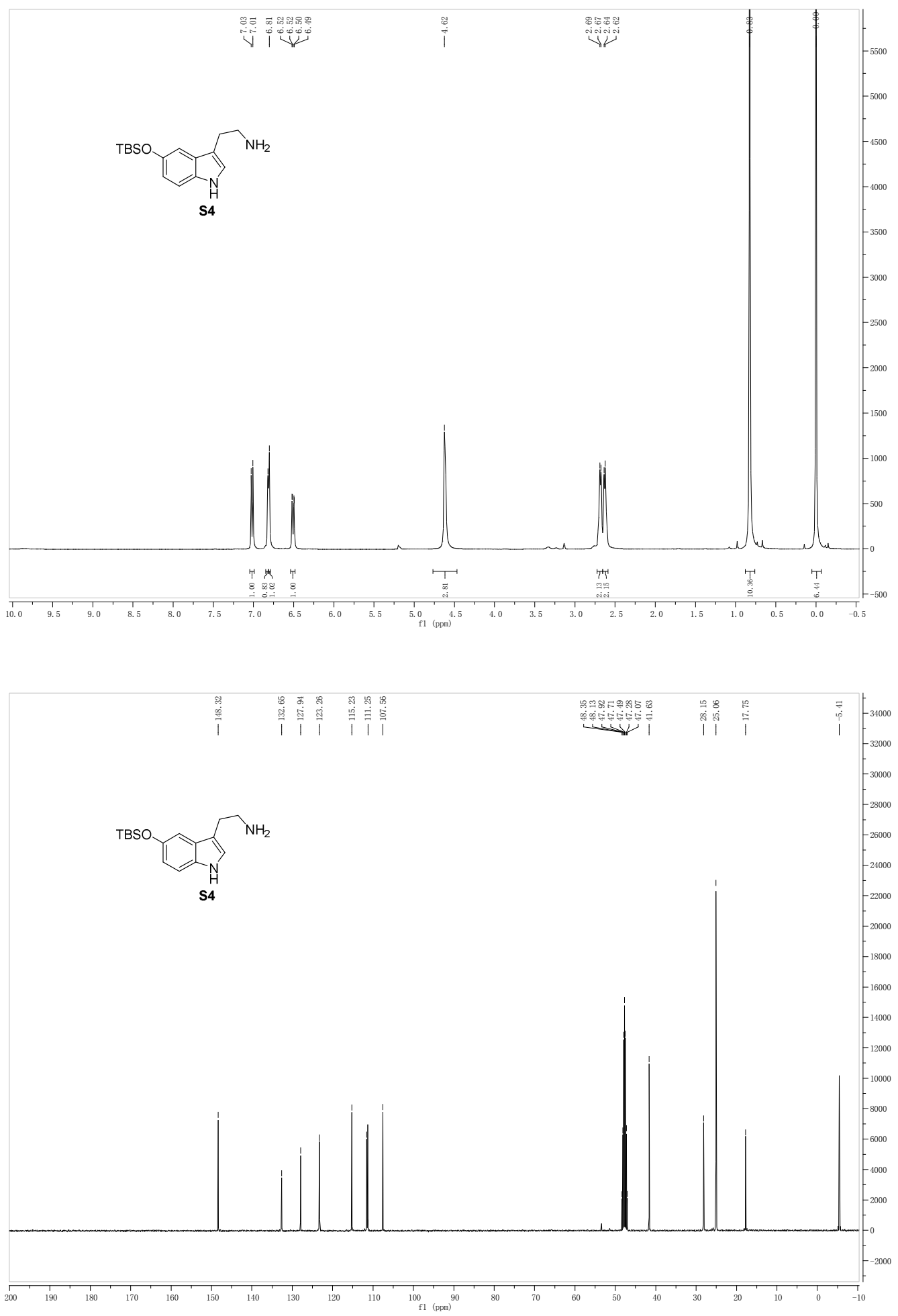

Supplementary Figure 23. ${ }^{1} \mathrm{H}$ and ${ }^{13} \mathrm{C}$ NMR spectra for S4. 

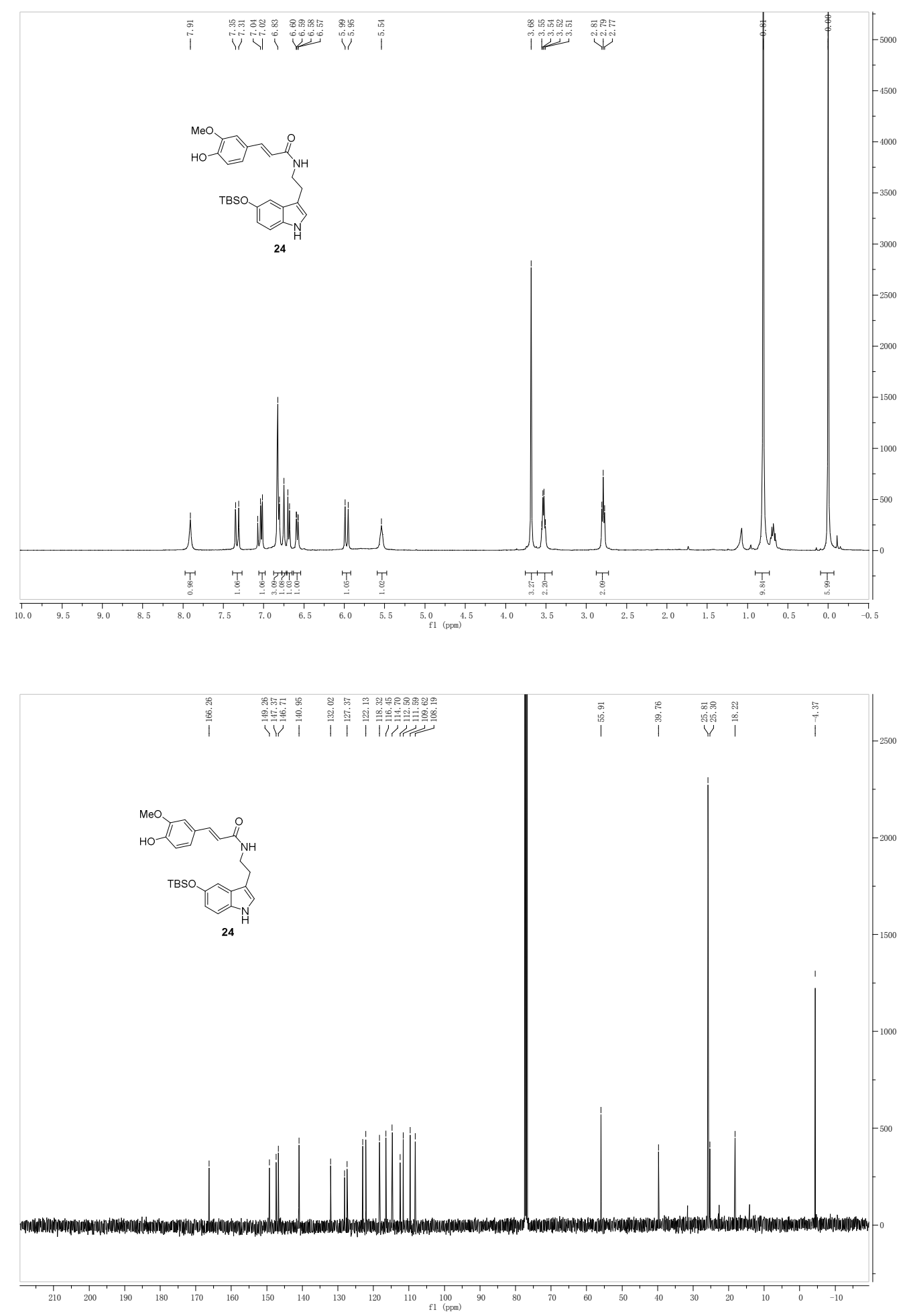

Supplementary Figure 24. ${ }^{1} \mathrm{H}$ and ${ }^{13} \mathrm{C}$ NMR spectra for 24. 

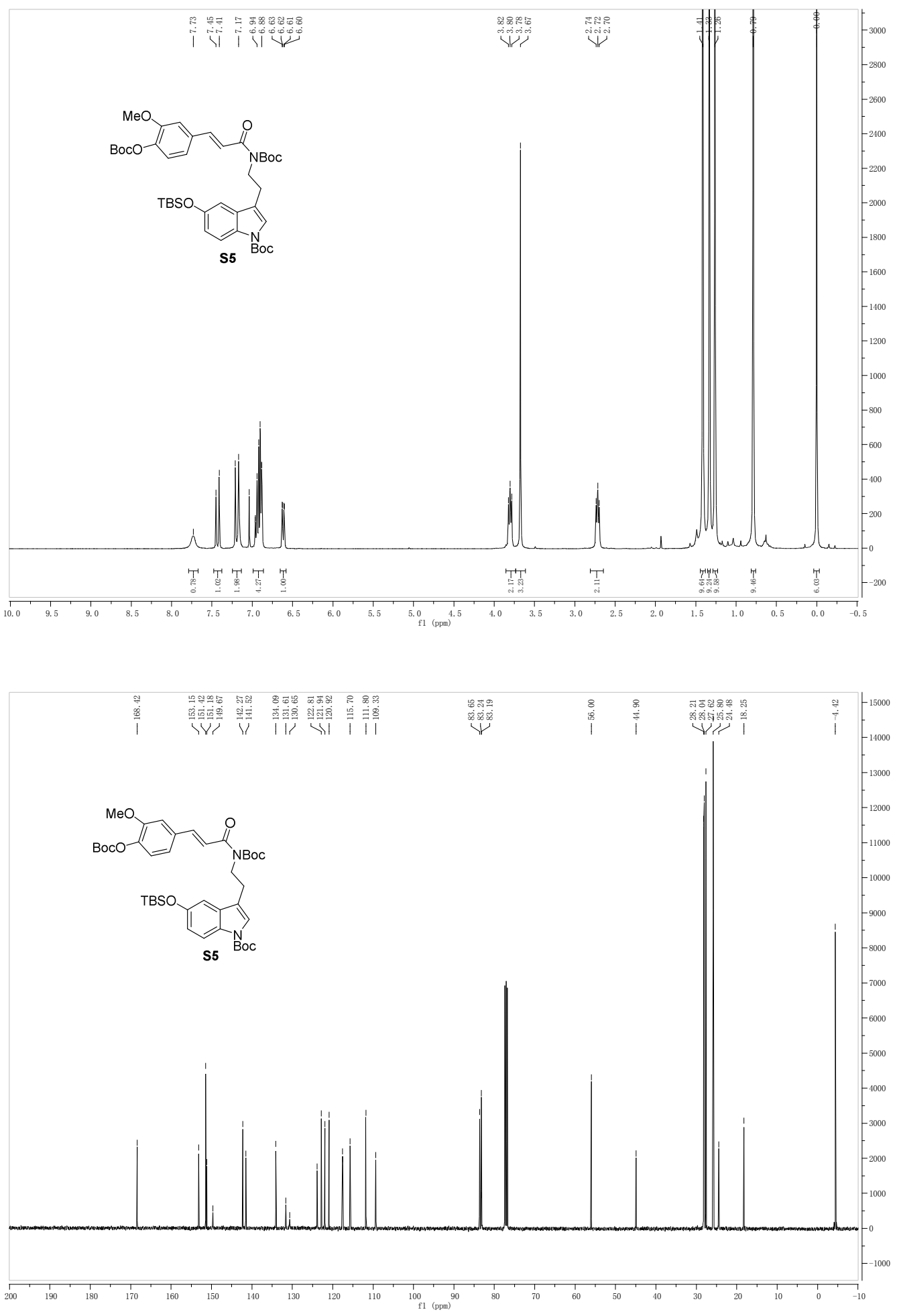

Supplementary Figure 25. ${ }^{1} \mathrm{H}$ and ${ }^{13} \mathrm{C}$ NMR spectra for S5. 

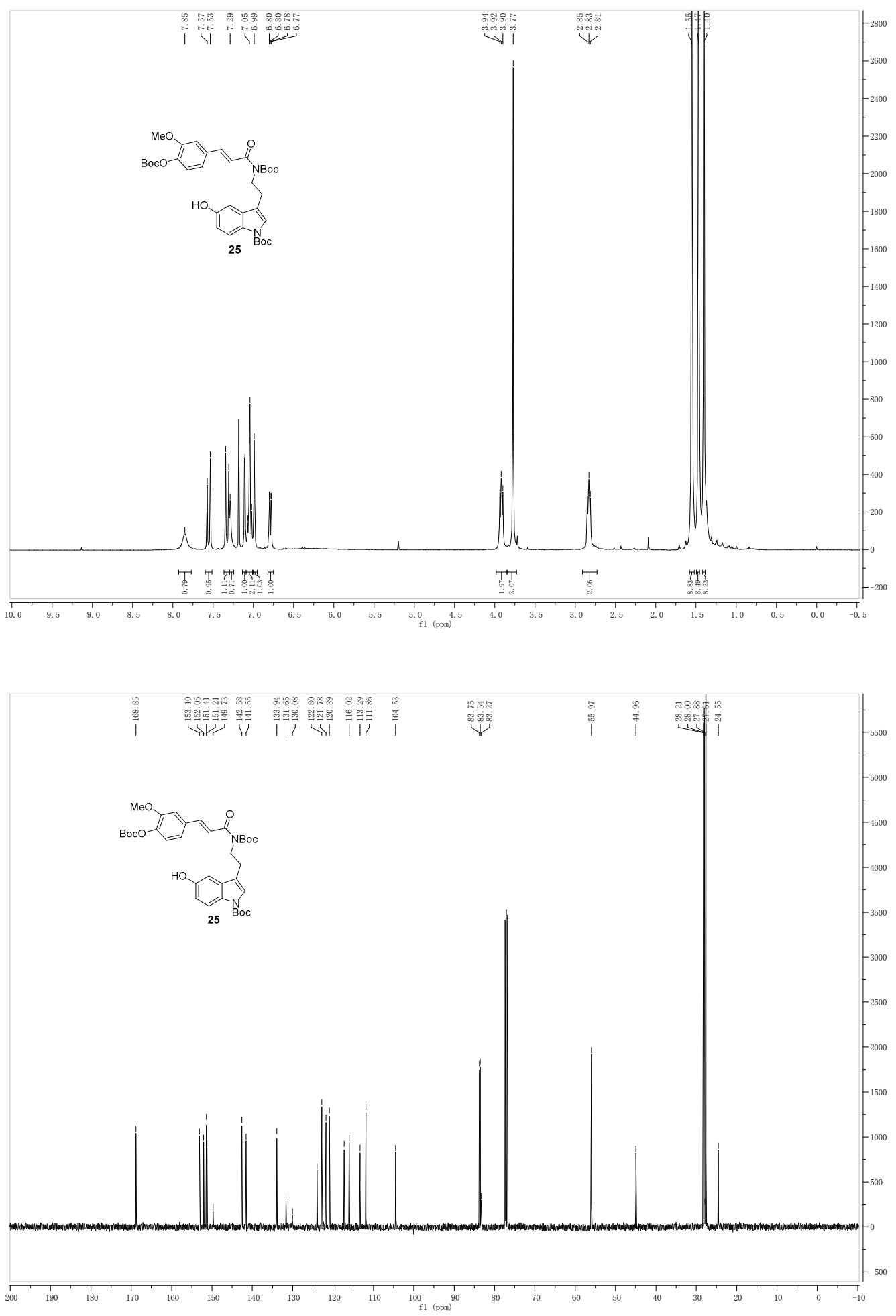

Supplementary Figure 26. ${ }^{1} \mathrm{H}$ and ${ }^{13} \mathrm{C}$ NMR spectra for 25. 

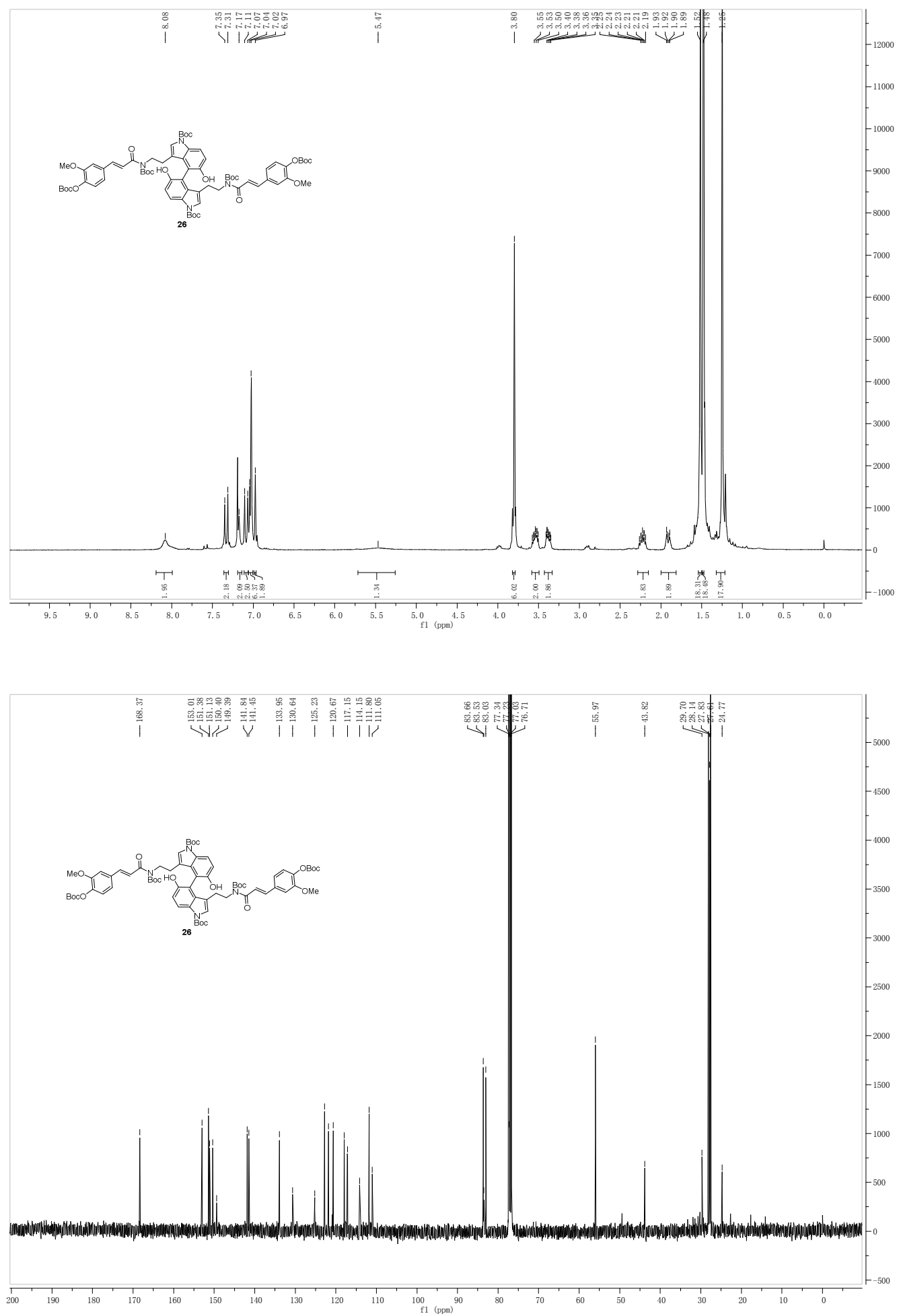

Supplementary Figure 27. ${ }^{1} \mathrm{H}$ and ${ }^{13} \mathrm{C}$ NMR spectra for 26. 

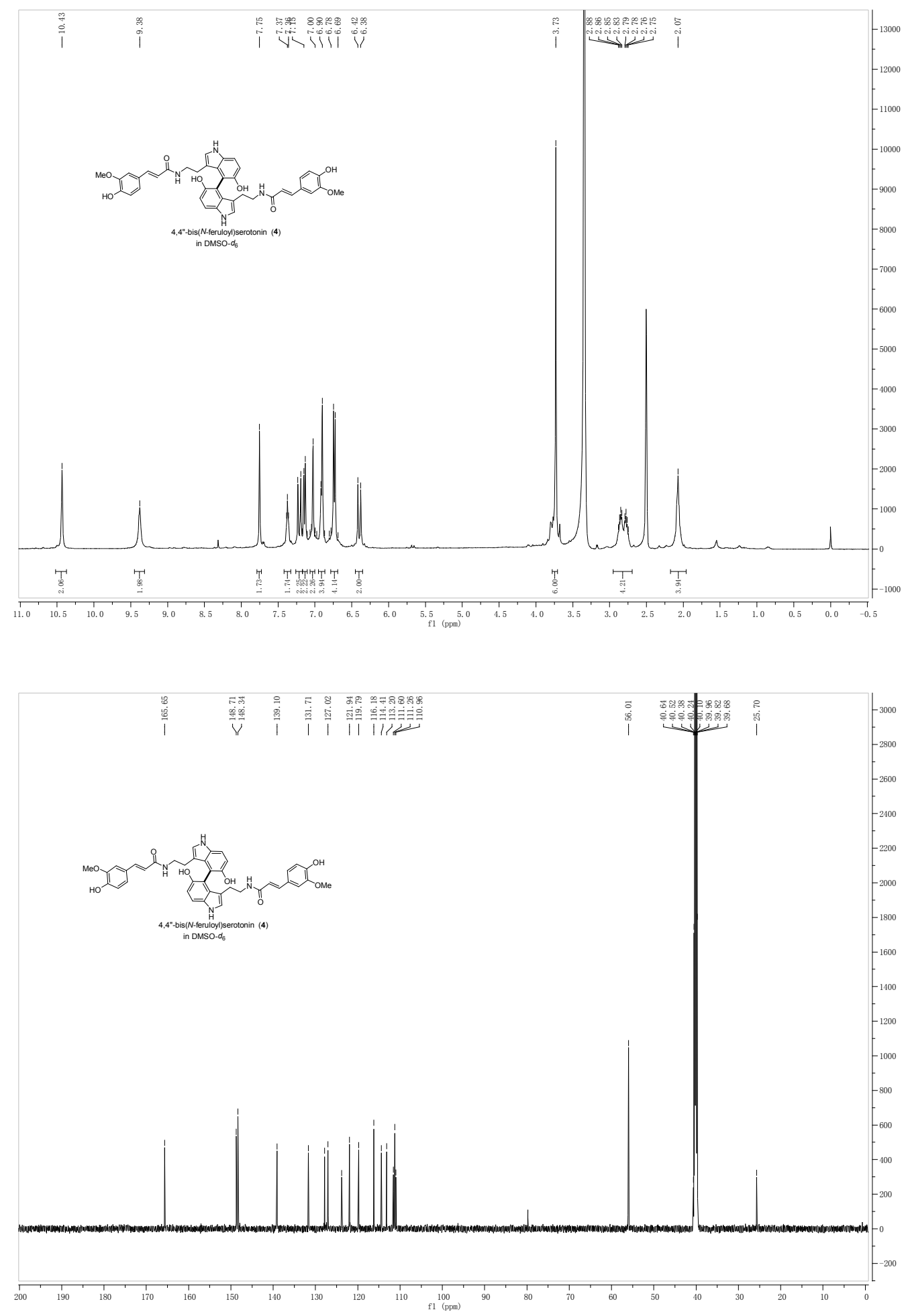

Supplementary Figure 28. ${ }^{1} \mathrm{H}$ and ${ }^{13} \mathrm{C}$ NMR spectra for $4,4^{\prime \prime}$-bis( $N$-feruloyl)serotonin (4) in DMSO-d6. 

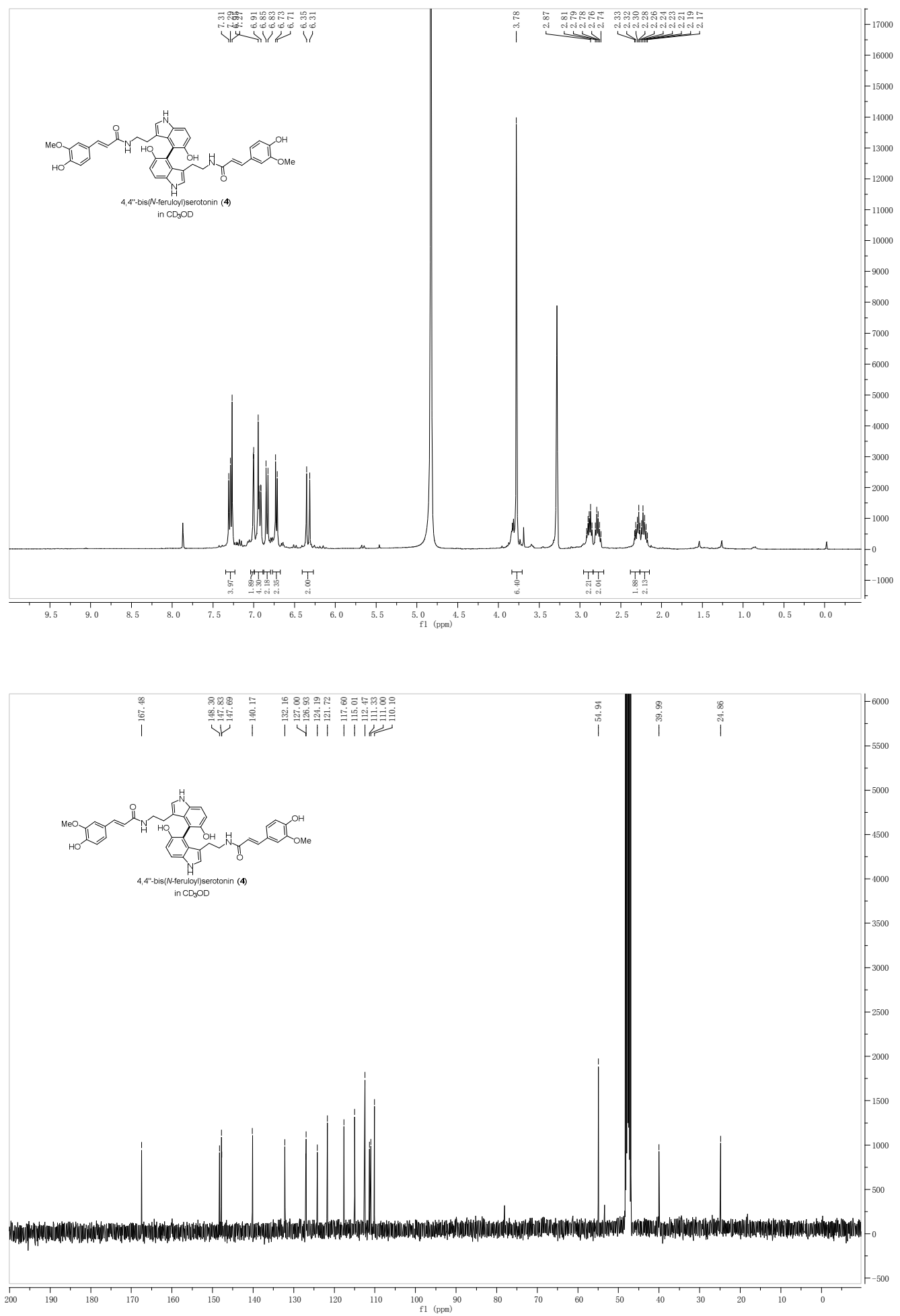

Supplementary Figure 29. ${ }^{1} \mathrm{H}$ and ${ }^{13} \mathrm{C}$ NMR spectra for $4,4^{\prime \prime}$-bis( $N$-feruloyl)serotonin (4) in $\mathrm{CD}_{3} \mathrm{OD}$. 